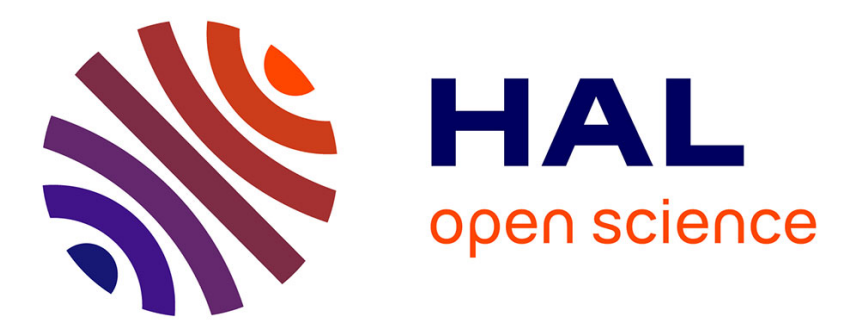

\title{
La vie pour l'épargnant ne se réduit pas à un exercice de calcul : Les apports d'une approche existentielle du cycle de vie (2)
}

André Masson

\section{- To cite this version:}

André Masson. La vie pour l'épargnant ne se réduit pas à un exercice de calcul: Les apports d'une approche existentielle du cycle de vie (2): Les apports d'une approche existentielle du cycle de vie. Revue française de l'Economie, 2010, 25 (2), pp.3-57. 10.3406/rfeco.2010.1804 . halshs-00754493

\section{HAL Id: halshs-00754493 \\ https://hal-pjse.archives-ouvertes.fr/halshs-00754493}

Submitted on 29 May 2020

HAL is a multi-disciplinary open access archive for the deposit and dissemination of scientific research documents, whether they are published or not. The documents may come from teaching and research institutions in France or abroad, or from public or private research centers.
L'archive ouverte pluridisciplinaire HAL, est destinée au dépôt et à la diffusion de documents scientifiques de niveau recherche, publiés ou non, émanant des établissements d'enseignement et de recherche français ou étrangers, des laboratoires publics ou privés. 


\section{André \\ MASSON}

\section{La vie pour l'épargnant ne se réduit pas à un exercice de calcul}

1) Les impasses des approches du cycle de vie standard et psycho-économique (paru dans le précédent numéro)

2) Les apports d'une approche existentielle du cycle de vie 


\section{Les apports d'une approche exis- tentielle du cycle de vie}

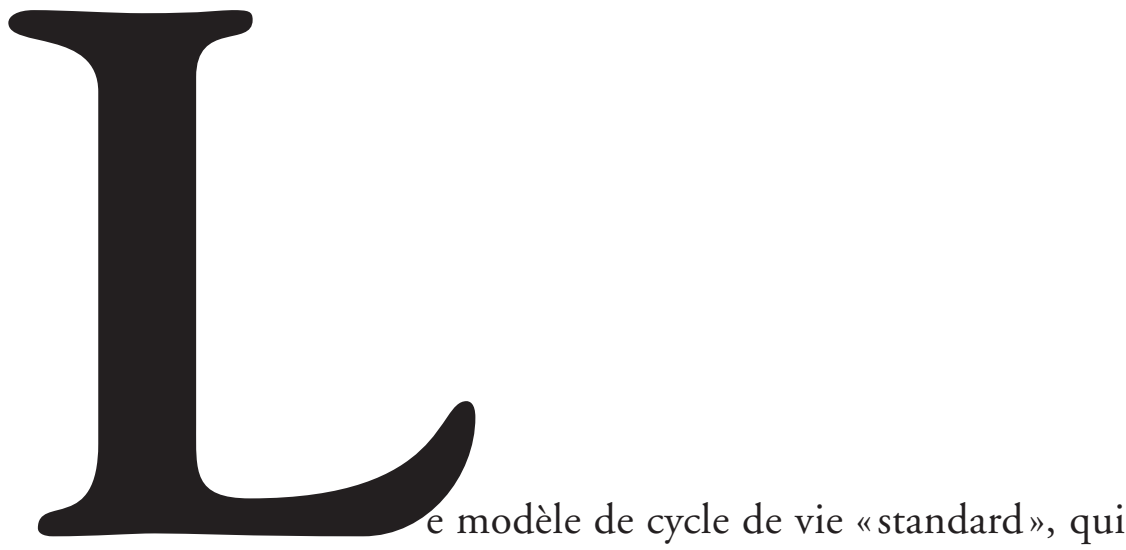
repose sur la maximisation d'une fonction d'utilité temporellement additive à actualisation exponentielle et suppose que les décisions en incertain sont régies par le critère de l'utilité espérée, aboutit à des prédictions radicalement contraires à l'observation en matière de comportements patrimoniaux, en particulier: (i) l'épargne - hors transmission - générée par des choix temporellement cohérents et prévoyants est " adéquate " aux besoins des vieux jours ; (ii) la quasi-totalité des ménages devraient posséder des rentes viagères, au moins après la retraite, même si une partie du patrimoine est réservée à la transmission intergénérationnelle : en raison de la mutualisation des risques de survie, la rente bénéfice en effet d'un rendement supérieur aux placements traditionnels (et cela en dépit des imperfections des marchés de l'assurance vie) ; (iii) en dépit, là encore, des coûts d'information et de transaction, la grande majorité des ménages devrait détenir des actions - fût-ce pour des montants limités - en raison du rendement élevé de ce placement risqué à long terme.

Or, l'observation montre qu'au moins un quart des ménages en France ou aux Etats-Unis n'épargnent pas assez pour leurs vieux jours, au risque de subir une diminution brutale de 
la consommation après la retraite, et que, plus généralement, la plupart des épargnants sont loin de toujours adopter des comportements temporellement cohérents, regrettant souvent leurs inconséquences passées (i). Un des défis les plus redoutables pour l'hypothèse du cycle de vie concerne par ailleurs la faible diffusion des rentes viagères dans la plupart des pays (ii). Enfin, dans des pays comme la France, l'Italie ou les Pays-Bas, la détention d'actions, directe ou indirecte (Sicav non monétaires, fonds communs de placement, etc.), concerne au plus un quart des ménages ; aux Etats-Unis même, seule la moitié de la population est concernée (iii).

Dans un article précédent (Masson, [2010]), nous avons rappelé comment une approche comportementale (behavioral) ou psycho-économique en plein développement s'est livrée à une attaque en règle contre l'irréalisme de ce modèle standard, en dénonçant les " anomalies" ou biais des comportements des épargnants : incapacité de planifier à long terme, "émotions" intempestives, biais de perception multiples, etc. Le problème vient de ce que cette approche semble imposer des limites très fortes à la rationalité de l'épargnant. Aussi ses explications des trois énigmes (i) à (iii) n'apparaissent-elles pas entièrement convaincantes parce qu'elles reposent in fine sur l'hypothèse d'épargnants par trop timorés ou myopes - sinon stupides.

L'approche "existentielle» développée ici attribue ces échecs relatifs du courant psycho-économique au fait que ce dernier fait l'impasse sur le " contexte situationnel " dans lequel se trouve présentement l'épargnant et qui conditionne ses choix de long terme : en affirmant que le sujet ne peut toujours adopter un point de vue totalement extérieur et objectif par rapport à sa vie, elle s'efforce de prendre en compte sa subjectivité proprement humaine qui s'exprime dans les rapports de soi à soi dans le temps et dans les projets conférant aujourd'hui sens et substance à son existence ; elle admet que la continuité de la vie ne va pas de soi, en insistant sur les ruptures qui scandent l'horizon temporel aux nouds ou bifurcations du cycle de vie, qu'ils soient subis (maladie, veuvage) ou qu'ils constituent des décisions clefs du sujet (mariage, enfant...) ; et elle reconnaît que ses rapports à la 
mort et à la finitude ne sont pas seulement régis par les probabilités mathématiques de survie.

Pour rendre compte de certaines anomalies et des hétérogénéités des comportements patrimoniaux sans forcément taxer d'irrationnels ou d'incompétents les épargnants, l'approche existentielle reposerait ainsi sur trois piliers : le primat du contexte situationnel présent, d'où partent des décisions structurées autour du projet en cours (mariage, métier, acquisition du logement, étude des enfants, retraite, etc.) ; la subjectivité des rapports de soi à soi qui dépend de la force et de l'étendue de ce projet actuel et des bifurcations choisies ou subies au cours de l'existence ; l'idiosyncrasie de l'attitude individuelle à l'égard de la mort, qui dépend des projets de vie poursuivis et se situe ainsi dans un rapport plus ou moins étroit avec l'espérance de vie.

Or, le formalisme des modèles du cycle de vie permet d'incorporer sommairement ces éléments par le canal de la préférence pour le présent, à condition de la doter d'une interprétation nouvelle : le moi à venir en $t$ verra son utilité affectée d'un poids ou facteur d'actualisation temporelle qui dépendra de la nature, de la force et de l'étendue des projets que le moi présent a pour lui, des raisons de vivre qui le concernent - et qui font que ce moi présent se soucie à des degrés variables de ces répliques plus ou moins lointaines de lui-même, s'identifie plus ou moins à ces dernières. Ce facteur d'actualisation pourra présenter des sauts de discontinuité aux nœuds de l'existence, choix de vie ou coups du sort qui rythment le déroulement de l'existence individuelle ou familiale et fragmentent l'horizon $\mathrm{du}$ sujet en autant de phases du cycle de vie. Et la préférence temporelle dépendra encore de l'attitude subjective du sujet à l'égard de sa propre mort : l'idée que cette relation ne se réduit pas à une distance temporelle réglée par un critère d'utilité espérée, appliqué aux probabilités mathématiques de survie, sous-tend déjà certains développements récents du courant psycho-économique (Masson, [2010]).

En s'appuyant sur les leçons générales de la philosophie du temps et de l'identité personnelle, l'approche existentielle éloignerait ainsi les choix de vie de l'épargnant d'un simple 
exercice de calcul ; en adoptant une conception plus large et plus subjective de sa rationalité, elle permettrait de mieux répondre aux énigmes (i) à (iii) que soulèvent ses comportements.

Cette approche met notamment en lumière les déboires auxquels s'expose l'économie expérimentale lorsqu'elle vise à reproduire le déroulement du temps de la vie in vitro, en sortant les individus-cobayes de leur contexte situationnel actuel pour les placer dans des conditions artificielles (à l'instar d'étudiants transformés en célibataires de 35 ans appelés à vivre jusqu'à 75 ans...) ; or les critiques psycho-économiques sur les préférences des épargnants à l'égard du temps - dénonçant à l'envi leur incapacité de se projeter dans l'avenir, leur propension à planifier trop limitée, leur imprévoyance et l'insuffisance de leur épargne pour la retraite - sont fondées en priorité sur les résultats problématiques de ces expériences de laboratoire.

L'approche existentielle avance par ailleurs deux facteurs explicatifs de la relative désaffection pour la rente viagère : un horizon décisionnel de l'épargnant dominé par l'échéance du projet en cours et inscrit dans le temps morcelé qui sépare les phases successives du cycle de vie ; un rapport à la mort qui ne se limite pas à la considération des seules probabilités mathématiques de survie (qu'elles soient correctement anticipées ou non).

La diffusion limitée des actions pourrait enfin s'expliquer par le terme trop rapproché du projet en cours, sa nature et ses objectifs (autres que la liquidité ou le rendement), mais aussi par le type de risques déjà supportés, qui ne sont pas seulement financiers : l'échec possible du projet que l'épargnant a particulièrement à cœur (tel que l'accession à la propriété) le confronte en effet à un risque "existentiel ", l'incitant à s'exposer d'autant moins par ailleurs. L'importance variable de ce risque existentiel, qui dépend beaucoup de la nature et des spécificités du projet actuel, permettrait en outre de comprendre pourquoi les retraités, pourtant plus averses au risque, ont une probabilité d'investir en bourse autant, sinon plus élevée que les autres ménages. 


\section{Notations (formalisme standard)}

Commençons par rappeler la structure des préférences dans le modèle de cycle de vie standard, en adoptant les mêmes notations que dans le texte précédent (Masson, [2010]).

Dans la version la plus simple, l'épargnant ne retire de satisfaction que des volumes globaux de consommation à chaque période de son existence, soit $C(t)$ à l'âge $t$. Dans un monde certain, il maximise à la date $s$ une utilité additive actualisée jusqu'à sa mort en $T$ :

$$
U_{s}[C(s) \ldots C(T)]=\int_{t=s}^{T} \alpha(t) u[t, C(t)] d t, \quad \alpha(t) \geq 0
$$

où le paramètre $\alpha(t)$ représente le facteur d'actualisation temporelle, soit la pondération décroissante accordée au flux d'utilité instantanée $u(t$..) du fait de l'existence d'une préférence pour le présent. Le taux de dépréciation du futur $\delta(t)$ est, au signe près, sa dérivée logarithmique :

$$
\begin{gathered}
\delta(t)=-\frac{d \alpha(t) / d t}{\alpha(t)}=-\frac{\alpha^{\prime}(t)}{\alpha(t)} \geq 0 ; \\
\alpha(t)=\exp \left(-\int_{0}^{t} \delta(t) d t\right)=\exp (-\delta t) \text { si } \delta(t)=\delta
\end{gathered}
$$

Un taux d'actualisation $\delta$ constant conduit à une actualisation exponentielle; plus $\delta$ est élevé, et plus l'horizon décisionnel de l'agent est court. Les choix sont temporellement cohérents.

Les préférences étant supposées homothétiques, la fonction d'utilité instantanée $u$ est de forme invariante et puissance :

$$
u\left[t, C(t]=u[C(t)]=C(t)^{1-\gamma} /(1-\gamma), \quad \gamma>0, \quad \gamma=1: u=\log C\right.
$$

où $\gamma$ représente le degré de concavité de $u$ ou encore l'élasticité de l'utilité marginale décroissante $\left(u^{\prime}=C^{-\gamma}\right)$. Avec des marchés des capitaux parfaits, la consommation à l'instant $t$ s'écrit :

$$
C(t)=k(t ; r, T, \delta, \gamma) W(t),
$$


où $W(t)$ représente le montant de ressources disponibles à la date $t$, somme de l'actif net et des revenus du travail présent et futurs, actualisés au taux d'intérêt $r$, et $k(t)$ la propension marginale à consommer la richesse en $t$, soit la valeur d'annuité de la richesse : $k(t)$ vaut $1 /(T-t)$ si $r=\delta=0$. Le taux de croissance de la consommation, $g(t)$, vérifie alors la relation :

$$
g(t)=[d C(t) / d t] / C(t)=\sigma[r-\delta(t)] \quad \sigma=1 / \gamma>0
$$

où $\sigma$ représente l'élasticité intertemporelle de substitution (ou l'élasticité de la consommation au taux d'intérêt $r$ ) ; $\gamma$ mesure à l'inverse le désir de lissage intertemporel de la consommation.

Dans un monde incertain, l'épargnant maximise l'espérance de son utilité, si bien que le degré de concavité de l'utilité instantanée $\gamma$ s'interprète également comme l'aversion relative à l'égard du risque, $\rho\left(=-C u^{\prime \prime} / u^{\prime}\right)$. On obtient finalement les égalités suivantes, caractéristiques du modèle standard :

$$
\gamma=1 / \sigma=\rho
$$

Le formalisme standard est donc très parcimonieux puisqu'il ne requiert que deux paramètres de préférence exogènes et " orthogonaux " : un seul, le taux de dépréciation du futur $\delta$, gouverne les choix par rapport au temps; et un seul, $\gamma$, détermine tous les comportements à l'égard du risque (prudence, tempérance, etc.).

En particulier, face à l'incertitude de sa durée de vie, avec $S(T)$ sa loi de survie - telle qu'il l'anticipe à l'instant initial $(t=0)$-, l'épargnant maximise l'espérance de son utilité qui prend la forme suivante (Yaari [1965]) :

$$
E U(C)=\int_{t=0}^{T m} \alpha(t) S(t) u[C(t)] d t
$$

où $C$ désigne encore le profil de consommations anticipées et Tm représente la durée de vie maximale possible (proche de 120 ans) $: S(T m)=0$. Le facteur d'actualisation temporelle est le produit $\alpha(t) S(t)$, correspondant à un taux de dépréciation du futur égal à :

$$
\delta(t)+q(t), \quad \text { avec }: \quad q(t)=-S^{\prime}(t) / S(t)
$$


$q(t)$ étant le quotient instantané de mortalité, dérivée logarithmique de $-S(t)$.

Supposons que l'épargnant puisse transformer son patrimoine en une rente viagère actuariellement neutre : celle-ci aura pour rendement $r+q(t)$, si bien que son profil de consommation, d'après la relation (5), ne dépendra plus des taux de mortalité (Yaari, [1965]). S’il ne peut ou ne veut recourir à la rente viagère, l'épargnant va laisser derrière lui, à sa mort en $T$, un patrimoine $A(T)$ positif qui correspond, modulo ses probabilités de survie, à ce qu'il aurait consommé si Dieu lui avait prêté une vie plus longue, éventuellement jusqu'en Tm. Ce legs «accidentel» n'est pas déterminé par un motif de transmission avéré : il résulte de l'épargne de précaution contre le risque de se retrouver âgé et démuni.

L'épargnant peut encore avoir un véritable motif de transmission "altruiste " pour ses enfants, supposé séparable de l'épargne de cycle de vie, dont l'importance dépendra de son degré d'altruisme $\theta(n)$, supposé en général fonction croissante du nombre d'enfants $n$ (Masson, [2010]).

Au total, les comportements patrimoniaux de l'épargnant standard dépendraient tout au plus de trois paramètres de préférence indépendants : $\gamma$ pour le lissage intertemporel de la consommation et les attitudes à l'égard du risque $; \delta$ pour la prévoyance et l'horizon décisionnel ; et $\theta$ pour l'altruisme intergénérationnel.

\section{L'approche existentielle : subjectivité et finitude humaines}

S’appuyant d'abord sur des tests expérimentaux inspirés des travaux de psychologues, les attaques les plus dévastatrices de l'approche psycho-économique contre ce modèle portent sur les attitudes des épargnants à l'égard du temps : incohérence tem- 
porelle, imprévoyance ou myopie, propension à planifier limitée ou même incapacité à se projeter dans l'avenir.

Portant précisément sur ces matières, l'approche existentielle entend prendre au sérieux les enseignements des philosophies " anti-idéalistes » de la temporalité de l'existence et de l'identité personnelle ${ }^{1}$ à savoir, les spécificités du mode d'être de l'homme et d'une subjectivité humaine qui s'exprime dans les rapports idiosyncratiques de l'individu au temps, «berceau " de cette subjectivité (Merleau-Ponty, [1945]). L'épargnant ne gère pas sa vie comme le ferait le manager d'une grande entreprise qui doit seulement contrôler ses émotions, et le point de vue qu'il manifeste à l'égard de sa propre mort differe de celui qu'aura l'actuaire. Bref, le "contexte situationnel» dans lequel il se trouve fait que ses choix fondamentaux ne se limitent pas à un pur exercice de calcul comme le voudrait l'économiste ou le professionnel.

Une brève incursion dans le domaine philosophique ${ }^{2}$ permettra de montrer qu'une approche existentielle de l'être humain conduit à élargir le champ de sa rationalité au-delà de ce que voudrait l'approche psycho-économique, et cela sur deux plans essentiels : en proposant une conception nouvelle de la préférence pour le présent, discontinue aux nœuds événementiels de l'existence ; en montrant que les rapports qu'entretient un épargnant pleinement rationnel avec sa propre fin ne se limitent pas forcément à ceux déterminés par ses probabilités de survie.

\section{Contre la conception rawlsienne de la temporalité de l'existence et de l'identité personnelle}

L'idée directrice est que le sujet ne peut guère envisager son existence d'un point de vue extérieur, comme la réalisation d'un plan d'un seul tenant qui la couvrirait toute entière. Le plus souvent, il ne peut se placer en "position originelle» par rapport aux différents moments de sa vie comme le recommande Rawls [1971], qui prétend que: «nous devons considérer notre vie comme un tout, comme les activités d'un sujet rationnel étalées dans le temps; il faut que nous prenions conscience de nous- 
mêmes comme d'une seule personne identique à travers le temps pour éviter d'avoir plus tard à regretter nos actions ou à nous adresser des reproches; [nous devons trouver ainsi] le projet qui organise nos activités et qui influence la formation des désirs qui en découlent au mieux de nos buts et de nos intérêts. "

Les tenants de l'approche psycho-économique, comme nombre d'économistes ou de professionnels, partagent implicitement cette conception rawlsienne d'un sujet totalement transparent et identique à lui-même, dont la vie est assimilée à un cadre ou " rectangle déterminé à l'avance qui devrait seulement être rempli optimalement $\gg .^{3}$

Les philosophies anti-idéalistes dénoncent cette assimilation du sujet à une conscience absolue "survolant de très haut l'existence et ses possibilités " (Heidegger, [1927], \$ 62) et soulignent au contraire l'opacité et l'altérité des rapports du moi présent avec ses moi futurs. Les décisions du sujet partent du présent et sont conditionnées par les rapports de soi à soi dans le temps, "horizon de la compréhension de l'être ». Corrélativement, la continuité de la vie n’est pas une "réalité-déjàdonnée " mais une œuvre : la cohésion (Zusammenhang) de mon existence dépend de mes choix, des projets du moment qui lui donnent sens et substance aujourd'hui.

\section{Du temps du calcul à la temporalité " authentique " (Heidegger)}

Un détour par l'analyse heideggerienne du temps humain éclaire le saut accompli par ces philosophies anti-idéalistes. Heidegger [1927] distingue, au-dessus de la représentation commune du temps donnée par les horloges, trois niveaux de temporalisation «d'authenticité» croissante.

"L'intra-temporalité» (Innerzeitigkeit) est le temps dans lequel on est et agit, "avec lequel il faut compter et donc calculer". Ce temps concret, quantité et outil, est celui que l'entrepreneur cherche à utiliser au mieux, celui de son agenda. Il differe du temps chronologique en ce qu'il constitue un temps unifié, confi- 
guré à partir de la fin, à la manière de l'intrigue d'un récit qui se comprend à partir de son dénouement (Ricoeur, [1990]). Parfaitement maîtrisable et transparent, c'est le temps rawlsien par excellence, qui fait de l'homme ni plus ni moins un animal rationale - un animal doué de raison - pour lequel la vie se réduit effectivement à un exercice de calcul ou de planification. Ce temps traduit bien les arbitrages quotidiens ou de courte portée, qui ne remettent pas en cause l'identité du sujet. Mais il ne convient pas pour les choix de vie où s'exprime la subjectivité humaine à travers le mode de perception de soi dans le temps, la nécessité de sauvegarder son intégrité psychique et de préserver ses raisons de vivre, ou la volonté de conférer un sens à son existence. Pour Heidegger [1946], il manque ainsi la spécificité de la manière humaine "d'être " qui prend la forme du "souci " (de soi), spécificité qu'expriment les niveaux de temporalisation supérieurs, introduisant à la structure " existentiale » du Dasein (l'être humain).

Le plus authentique est celui de la " temporalité " (Zeitlichkeit), qui désigne le mode de présence à soi dans l'instant vécu. Dans une optique prospective, le Dasein est alors celui pour lequel il y va toujours de lui-même, qui existe à dessein de soi, en fonction de ce qu'il a à être, qui est en quelque sorte sa propre fin (Heidegger [1927], \$12). Toujours déjà " jeté au monde ", il est tenu d'assumer son existence comme une tâche qu'il ne peut éluder. C'est un pouvoir-être, s'anticipant comme tel par le souci, au sens de prendre soin de l'existence à laquelle il est voué. La transcendance du Dasein n'est donc pas celle d'une substance, mais se fonde sur une intentionnalité : exister, c'est se temporaliser en se transportant hors de soi-même, se dépasser vers ses potentialités, saisir ou manquer ses propres possibilités jusqu'à la perspective inéluctable de la mort - entendue ici comme la "possibilité ultime ", celle de renoncer à soi-même.

La temporalité ne réalise cependant qu'une synthèse ponctuelle et précaire du Dasein, qui semble être toujours en "sursis " et "sautiller " d'un instant à l'autre de sa vie. Dans ce contexte, la cohérence ou l'unité de l'existence, que Rawls attribue naturellement à la permanence d'un caractère (i.e. à la stabilité des préférences), ne va plus de soi. Elle se joue précisément à la 
flexion entre les deux niveaux de temporalisation supérieurs, " historialité "(Geschichtlichkeit) et temporalité, dans le lien entre le passé et le projet (Heidegger [1927], \$ 74). La plus ou moins grande " constance à soi " (Selbständigkeit) du Dasein dépend de la capacité « à faire histoire " (Geschehen) et s'effectue par l'expérience de la répétition, qui est la « reprise de ses possibilités les plus propres, la répétition de soi-même comme destin [et consiste] à reconnaître son pouvoir-être dans son avoir-été ". S'il y a jamais de réel pour lui que l'instant vécu, l'être humain n'en est pas moins un projet qui peut assurer un haut degré de continuité de sa vie par la répétition résolue, en quelque sorte la fidélité à soi-même et à ses possibilités, assumée à la manière d'une promesse tenue (Ricoeur, [1990]). ${ }^{4}$

Si on l'arrête là en oubliant ses développements plus critiquables ${ }^{5}$, l'analyse heideggérienne apparaît tout à fait exemplaire des philosophies anti-idéalistes du temps de l'existence et de l'identité personnelle. C'est à la flexion entre historialité et temporalité que s'exprime le propre d'une subjectivité humaine clairement irréductible aux émotions de l'approche psycho-économique. La continuité et la cohésion de la vie dépendent de la nature, de l'extension et de la force des projets du moment, dans la mesure où ces derniers, précisément, donnent corps, sens et substance à l'existence aujourd'hui. Ces projets s'ancrent dans le présent parce qu'ils sont le fait d'un "sujet incarné et historique " et s'élaborent toujours sur la base d'un certain donné, s'appuient à chaque instant sur un «sol» constitué par le contexte situationnel actuel, le passé incorporé et la mémoire (MerleauPonty, [1945]). ${ }^{6}$

\section{La conception existentielle de la pré- férence pour le présent}

Cette analyse "existentiale " a le double mérite de fournir une justification intrinsèque à l'actualisation temporelle sur le cycle 
de vie tout en proposant une interprétation nouvelle, philosophique, du concept économique de préférence pour le présent $(\alpha<1)$. Dans le régime de l'intra-temporalité, i.e. des choix anecdotiques ou sans conséquence, il n'y a pas de raison impérieuse d'introduire une telle préférence (hors des considérations de survie) ; c'est bien la position qu'adopte Rawls en assimilant l'individu à un animal rationale et la vie à un exercice de calcul. Mais il n'en va pas de même pour les décisions de plus longue portée qui engagent l'identité du sujet et façonnent son destin : au niveau abstrait et opératoire de la modélisation, $\alpha(t)$ mesure sous forme scalaire le degré de continuité de l'existence, et (1- $\alpha(t))$ la plus ou moins grande opacité ou altérité du moi dans $t$ années par rapport au moi actuel. ${ }^{7}$

\section{Une préférence temporelle rationnelle discontinue, aléatoire et endogène}

Le premier économiste à avoir vraiment compris et défendu cette position est Von Mises [1949], le fondateur de l'école subjectivisme néo-autrichienne. Sa "praxéologie», ou théorie des actions intentionnelles, le conduit à inscrire la préférence pour le présent dans le temps du projet, comme une "composante catégorielle de l'agir humain [et donc pleinement rationnelle...] parce qu'il nous est impossible de penser une action sans vouloir sa réussite le plus tôt possible ». Pire, hors les vies édifiantes de quelques saints, tout entières vouées à une quête grandiose, l'absence de préférence pour le présent ne constitue pas un idéal, loin de là, mais correspond plutôt à " un tarissement pathologique de l'énergie vitale ", à l'exemple d'une avarice extrême.

A l'inverse, une préférence élevée pour le présent $(\delta>>0)$ ne découle pas forcément d'un manque de rationalité, mais traduira une myopie rationnelle, comportement temporellement cohérent qui peut à l'occasion se révéler auto-destructeur. Parfit [1984] donne l'exemple d'un jeune fumeur, parfaitement lucide et maître de lui, qui sait pertinemment les risques de santé qu'il encourt pour l'avenir mais ne s'en soucie guère, parce qu'il «ne 
s'identifie pas à son moi futur». Dans le modèle d'addiction rationnelle de Becker et Murphy [1988], les individus attirés par la drogue sont de même caractérisés par un taux élevé de dépréciation du futur.

Victimes d'une conception idéaliste de l'identité, les modèles économiques envisagent trop souvent le sujet dans le temps comme une série de moi réunis autour d'une table pour une délibération rationnelle, le seul privilège du moi présent étant qu'il décide le premier et peut ainsi contraindre les autres. La subjectivité humaine exige davantage : le moi en $t$ à venir voit son bien-être affecté d'un poids $\alpha(t)$ qui dépend de la prégnance et de l'étendue des projets que le moi présent a pour lui. Ce poids $\alpha(t)$ a métaphoriquement à voir avec sa survie (relation (8)) : le moi en $t$ " n'existe " pour le moi aujourd'hui que dans la mesure où ce dernier a des raisons de vivre qui le font se soucier de lui, des projets qu'il ne peut mener à bien sans lui. Le poids $\alpha(t)$ s'interprète aussi comme un degré d'auto-altruisme: il mesure l'intensité de la relation qu'entretient le moi présent avec cette réplique future de lui-même, la manière dont il se sent " concerné " par elle, l'empathie qu'il éprouve pour elle - ses possibilités d'identification avec cette dernière. Dans la mesure où les moi futurs s'apparentent à des "descendants " du moi présent, le paramètre $\alpha(t)$ s'inscrit encore dans une problématique de l'héritage : il dépend de la manière que l'on a de prendre en charge, d'assumer et de ressaisir, bref de transmettre à soi-même le lot de potentialités, de principes de vie ou de projets que l'on reçoit à chaque instant.

Quelles propriétés les enseignements des philosophies, mais aussi des sociologies anti-idéalistes, conduisent-ils à attribuer à la préférence temporelle dans le formalisme des modèles de cycle de vie (relations (1) à (5) notamment) ? Tout d'abord, il ne peut y avoir d'élan vers le futur projeté sans appui sur un " sol » pour parler comme Merleau-Ponty. Ces dimensions sociohistoriques et familiales de l'horizon temporel apparaissent déjà centrales chez des économistes comme Fisher [1930], qui fait dépendre le degré "d'impatience " des habitudes (familiales) et de la mode. Tout en préservant la cohérence des choix sur un 
horizon de terme $T$, le facteur d'actualisation $\alpha$ portera ainsi ces composantes qu'ignore autrement un comportement d'épargne standard purement autonome et prospectif. ${ }^{8}$

Mais surtout, la nouvelle conception de la préférence temporelle fait que $\alpha$ va présenter des sauts de discontinuité aux bifurcations ou ruptures du cycle de vie désirées ou subies, moments de vérité ou périodes de crise où s'éprouvent l'identité et la constance d'un sujet qui peut devenir autre après-coup ; ces nœuds événementiels, coups du sort, décisions en chaîne ou passages d'un projet fédérateur à un autre, scandent le déroulement de l'existence individuelle ou familiale et fragmentent l'horizon en autant de phases du cycle de vie (mariage, naissances, changement professionnel, départ des enfants, divorce, décès des parents et héritage, retraite, incapacité ou maladie grave, veuvage.... $)^{9}$. Comme le suggère Parfit ([1984], p. 328), ils peuvent être assimilés à de "petites morts", mais suivies de "petites renaissances", nouveaux départs dans l'existence où l'individu doit continuer à s'assumer. Le mariage - voir l'expression vieillie " enterrer sa vie de garçon "- ou la retraite, par exemple, s'apparenterait à un saut dans l'inconnu, petite mort puis petite renaissance où je vais (continuer à) découvrir ou inventer qui je suis.

Anticipées, les discontinuités peuvent concerner les deux éléments du couple $(\alpha, \delta)$. Notons $\alpha_{-}$et $\delta_{-}$les valeurs des paramètres avant l'événement considéré, $\alpha_{+}$et $\delta_{+}$après. L'écart $\left(\alpha_{-}-\alpha_{+}\right)$mesure l'importance subjective de la rupture ; un coup du sort malheureux - chômage, maladie, décès d'un proche - tend le plus souvent à diminuer les raisons de vivre, engendrant un $\delta+$ élevé (Becker et Murphy, [1988]) ; un événement «heureux" - mariage, enfant - élargit au contraire l'horizon (Fisher, [1930]), conduisant à une valeur de $\delta+$ beaucoup plus faible.

Ces moments clefs du cycle de vie conferent à la préférence temporelle un caractère à la fois aléatoire (state-dependent) et endogène. L'horizon de l'épargnant ne se confond plus avec la distance linéaire ou le temps homogène qui le sépare de la mort. Sa structure s'apparente à un arbre de décision déployant les 
trajectoires de vie possibles aujourd'hui selon les situations rencontrées et les choix effectués ${ }^{10}$. Le fait que $\alpha$ dépende de l'état de nature confere notamment une «tonalité» particulière à l'existence de l'individu. Une réinterprétation de l'Odyssée l'illustre clairement. Supposons qu'Ulysse ait bien pour but ultime de retrouver Pénélope sans laquelle sa vie n'aurait pas de sens : s'il apprenait la mort de sa bien-aimée, il n'aurait plus de projet dans l'existence et déciderait alors de faire une fin en se noyant avec les sirènes. Un futur totalement déprécié en cas de veuvage éventuel permet d'intégrer cette perspective. ${ }^{11}$

\section{Une rationalité élargie : myopie temporaire et mouvements d'accordéon de l'horizon}

Si cette interprétation de la préférence temporelle reproduit assez fidèlement certains traits cruciaux du " contexte situationnel " auquel sont confrontés les individus sur leur cycle de vie, quelles en sont les conséquences spécifiques sur la rationalité pratique des épargnants?

L'horizon décisionnel revêt maintenant un caractère dynamique, évoluant selon la position atteinte dans le cycle de vie et la trajectoire passée de l'épargnant. Procédant par étapes dans un environnement incertain, ce dernier hiérarchise les différents projets qui interviendront au cours de son existence en privilégiant ceux qui correspondent à ses préoccupations actuelles, qui donnent sens à sa vie aujourd'hui, en fonction de sa situation et de ce qu'il a déjà vécu. Jeune, il s'intéressera d'abord à l'acquisition du logement désiré pour lui-même et les siens, et peut ne s'engager dans une préparation "active " de sa retraite que beaucoup plus tard, aux âges mûrs, une fois les enfants partis et installés par exemple, lorsque la retraite apparaîtra le prochain événement (life event) à venir dans son programme de vie.

Surtout, la conception discontinue du temps humain engendre des myopies rationnelles temporaires : si les sauts de la préférence temporelle $\alpha$ sont importants, l'horizon semble en effet animé de "mouvements d'accordéon ", paraissant se boucher 
avant une échéance cruciale pour s'élargir à nouveau après-coup. Soit le cas anecdotique d'un étudiant au plan de carrière bien défini. En dépit de ses projets professionnels, ce dernier risque d'agir provisoirement comme un individu myope, obnubilé qu'il est par la fin de ses études - sorte de petite mort pour lui : la réussite de l'examen final ou de la thèse semble mobiliser toutes ses énergies et l'empêcher de voir au-delà. Son comportement est pourtant rationnel et temporellement cohérent : une fois l'échéance fatidique passée, ses choix de carrière apparaîtront effectivement guidés par une perspective de long terme.

Comment le formalisme standard (1)-(4) rend-il compte de cette myopie temporaire ? Grossissons le trait : l'étudiant est envisagé sur une période de 13 années, avec :

$$
\alpha(t)=1 \text { si } t<3 ; \alpha(t)=1 / 10 \text { si } 3 \leq t \leq 13
$$

Le profil de $\alpha(t)$ est en marches d'escalier : sur les trois années qui le séparent de la fin de ses études, l'étudiant est entièrement tendu vers la réalisation de cet objectif $(\alpha(t)=1)$, qui marque cependant un tournant dans son existence $(\alpha(t)=1 / 10$ après). Supposons un taux d'intérêt $r$ nul et une utilité instantanée logarithmique $(\gamma=1)$. La consommation suit alors le même profil selon l'âge que le facteur d'actualisation : $C(t)$ est proportionnel à $\alpha(t)$. L'horizon " empirique " de l'épargnant - pour parler comme Milton Friedman [1963] - est alors égal à $1 / \mathrm{k}(t)$, où $k(t)$ désigne la propension marginale à consommer la richesse ${ }^{12}$. Dans le cas présent, cet horizon vaut :

$$
1 / k(t)=\frac{1}{\alpha(t)} \int_{s=t}^{13} \alpha(s) d s
$$

il diminue de 4 à 1 avant $t=3$, puis remonte brusquement à 10 avant de décroître à nouveau pour s'annuler en $t=13$, présentant bien les mouvements d'accordéon décrits précédemment.

Au-delà de ce cas d'école, on voit que ces fragmentations de l'horizon élargissent sensiblement la rationalité de l'épargnant. Plus encore que les myopies rationnelles à $\delta$ constant, les myopies temporaires raccourcissent son horizon et peuvent donc justifier le rejet - fût-il provisoire - de placements rentables seulement à 
long terme, telles les rentes viagères ou les actions : le projet actuel est de trop courte portée pour que leur détention devienne intéressante.

En outre, l'épargnant peut tirer parti de ces fractures en les provoquant ( $\alpha$ endogène). Soit l'exemple tiré de Gary Becker [1992], dont on oubliera les connotations moralisatrices : «un jeune homme troublé [troubled], qui commence à se droguer, peut espérer, mais ne peut être certain, que sa vie va se redresser [straighten out] par un bon mariage ou un bon métier, avant de devenir toxicomane [addicted].» Selon la théorie beckerienne de l'addiction rationnelle, le jeune homme est attiré par la drogue parce qu'il a un taux de préférence $\delta$ élevé pour le présent, le rendant sujet aux comportements auto-destructeurs. Le métier ou le mariage entraînerait une baisse importante du facteur d'actualisation $\alpha$ (le sujet devient "autre " après un tel événement) mais aussi une diminution importante de son taux de dépréciation du futur $\delta$ après coup (sa vie "se redresse »), impliquant l'arrêt de la drogue : le jeune homme connaîtrait le plaisir sans la peine... ${ }^{13}$

Un dernier mot sur la prédictibilité des comportements. Selon l'interprétation proposée, la préférence temporelle $\alpha$, supposée a priori rationnelle, de chacun est directement conditionnée par son curriculum vitae (génétique, familial, professionnel, sociologique, culturel, etc.), en partie observable. Reste que cette préférence relève d'un "ordre plus élevé ", guidant des choix identitaires, qui porte sur les fins elles-mêmes et forge un caractère, un style de vie. Manifestant la subjectivité la plus propre de l'individu, elle relève de l'exercice de sa liberté personnelle (Merleau-Ponty, [1945]), et dépend de désirs "catégoriques", de raisons de vivre idiosyncratiques. Ce pourquoi elle n'est pas totalement prédictible.

Soit le cas d'un sujet en pleine possession de ses moyens, qui apprend soudainement qu'il est condamné avant 6 mois. Il affronte une crise existentielle aigüe qui l'oblige à reconsidérer tous ses projets, à se reconstruire, à redonner du sens au peu de vie qu'il lui reste. Il peut décider de faire la fête, dépenser inconsidérément, se permettre ou s'offrir tout ce qu'il n'a pas pu ou 
osé faire jusqu'alors, profiter au maximum du maigre temps imparti : son taux $\delta$ de dépréciation du futur croît à l'infini (recherche du plaisir immédiat). Mais il peut aussi bien être abattu, sans ressort, et se replier sur lui-même en adoptant une fin de vie austère : le présent n'ayant plus de prix, son taux $\delta$ tendra à s'annuler ${ }^{14}$. La position qu'il adoptera entre ces deux extrêmes dépendra certes de son histoire personnelle ; mais, ex ante, elle demeure en partie indéterminée.

\section{L'approche existentielle et le rapport à la mort}

Cet exemple fait la transition avec l'autre point clef de l'analyse existentielle, à savoir le rapport idiosyncratique de l'individu à sa mort, domaine par excellence de la philosophie qui le traite souvent sous le chapitre plus large du rapport de l'être humain à sa " finitude ». Les mêmes principes que précédemment s'appliquent encore (de fait, la mort correspond à $\alpha=0$, désormais) : par rapport à sa propre fin, le sujet ne peut en général adopter le point de vue objectif et purement statistique de l'assureur ou de l'actuaire, en termes de probabilités mathématiques de survie; en particulier, il ne peut suivre jusqu'au bout la rationalité standard qui conduit au critère d'utilité espérée (7) à la Yaari.

Pour chacun, la mort n'est pas seulement inéluctable et immanente. Elle est aussi radicalement incertaine (au sens que F. Knight donne à ce terme) - « son heure n'est pas venue » - tout en présentant un caractère endogène, dans la mesure où elle n'échappe pas totalement à notre contrôle, conscient ou non (ne serait-ce que par le souci apporté à notre santé). Retirer ces deux aspects cruciaux de la mort occulte des aspects essentiels de la condition humaine; cela reviendrait à me plonger dans un monde où ma mort serait programmée (supposons) le 14 janvier 2019 à 17 heures 30 précises, et cela sans que je ne puisse rien 
y changer (en sautant demain du haut de la Tour Eiffel, par exemple). Ce monde où ma vie se déroulerait comme un compte à rebours, telle celle d'un condamné à mort, se révélerait sans doute un enfer ; mais il serait surtout étranger au nôtre, et les comportements d'épargne y seraient fort différents.

Ajoutons par ailleurs que, comme toute préférence pour le présent, la perspective de la mort est ce qui fait le " prix » ou le sel de l'existence. Couplée à un traitement impartial des différents instants du temps $(\delta=0)$, l'immortalité $(T=+\infty)$ se révélerait d'un terrible ennui puisque l'action pourrait toujours être remise sans dommage au lendemain. Borges a commis de belles variations sur ce thème dans sa nouvelle intitulée précisément l'Immortel. Ou, pour évoquer une autre référence, soit la conférence de Jacques Lacan à Louvain le 13 octobre 1972. ${ }^{15}$ "La mort... est du domaine de la foi. Vous avez bien raison de croire que vous allez mourir, bien sûr. Ça vous soutient! Si vous n'y croyiez pas, est-ce que vous pourriez supporter la vie que vous avez ? Si on n'était pas solidement appuyé sur cette certitude que ça finira... est-ce que vous pourriez supporter cette histoire? Néanmoins, ce n'est qu'un acte de foi..."

\section{Les tentatives de modélisation récentes pour intégrer le rapport subjectif de l'épargnant à sa mort (résumé)}

Dans le texte précédent (Masson, [2010]), nous avons montré que des modèles récents ouvrent pour nous une voie prometteuse en soulignant l'importance du rapport subjectif du sujet à sa propre mort pour comprendre les énigmes que posent les comportements d'épargne.

En cela, ils ne font que reprendre et développer les intuitions de d'Alembert dans sa querelle des années 1760 avec Bernoulli sur l'opportunité de l'inoculation de la variole, ancêtre de la vaccination. L'inoculation comportait un risque de mort à court terme limité mais non négligeable ; en contrepartie, elle écartait le risque, beaucoup plus élevé mais éloigné, de périr ultérieurement de la maladie. Pour Bernoulli, qui adopte le 
pointe de vue impartial et objectif de la puissance publique (ou aussi bien celui de l'assureur " bienveillant»), la pratique doit être généralisée : fondé sur une anticipation de la relation (7), son calcul probabiliste, mené sur les tables de mortalité observées, montre qu'elle sauve des milliers de vie. Mais d'Alembert, qui se place du côté de l'individu, ou du père qui ferait inoculer ses enfants, exprime des doutes, proposant d'encourager le développement de cette pratique mais de ne pas l'imposer, pour au moins trois raisons qui nous intéressent ici :

- la première, aujourd'hui classique, vient de l'actualisation temporelle engendrée par la préférence pour le présent : le gain lointain, en termes d'années supplémentaires de vie, est certes beaucoup plus important, mais il est déprécié par rapport au risque proche de décès ;

- la deuxième met en balance le gain de l'inoculation en terme d'espérance de vie avec l'augmentation du risque affectant la durée de vie, à travers la probabilité accrue d'une mort précoce : traduite en termes actuels, l'idée correspond à une utilité intertemporelle qui est une fonction concave des satisfactions apportées par les années de vie successives ; l'application du critère d'utilité espérée implique alors une aversion au risque sur la durée de vie $;^{16}$

- la troisième met en avant la nature irréversible, unique et incertaine de la mortalité aux yeux de chacun : autrement dit, dans les décisions qui engagent leur existence, les individus se contentent rarement d'envisager leur rapport à leur fin sous l'angle purement objectif des probabilités mathématiques de survie et du critère d'utilité espérée ; la mort comporte toujours une part d'inconnu et peut notamment faire l'objet de déni, de refoulement ou d'angoisse, tous éléments que l'on peut difficilement qualifier d'emblée d'irrationnels (Masson, [2010]).

Les modèles récents en question ont cherché de même à séparer le point de vue objectif de la puissance publique ou de l'assureur (Bernoulli) et celui subjectif de l'épargnant sur son cycle de vie (d'Alembert). Ils ont cherché à rendre compte de la subjectivité humaine des rapports à la mort par le canal de la 
préférence pour le présent, en s'appuyant à chaque fois sur une version modifiée de la relation (8).

Bommier [2008] propose une formalisation rigoureuse du deuxième argument de d'Alembert et de la notion d'aversion au risque sur la durée de vie. Il introduit une fonction $\phi$ croissante et concave de l'utilité intertemporelle à durée de vie $T$ donnée, avant de prendre son espérance (selon la loi de survie $S$ ). La concavité de $\phi$ génère une préférence temporelle endogène pour le présent : mieux vaut consommer davantage aujourd'hui pour diminuer le risque d'une vie courte et "monotone " (i.e. de faible niveau de consommation). Cette préférence pour le présent augmente avec l'aléa de la durée de vie et le risque de mort prématurée. Comme la croissance historique de la longévité s'est d'abord accompagnée d'une orthogonalisation des lois de survie, l'horizon temporel des agents (leur prévoyance) aurait augmenté plus vite encore que l'espérance de vie, ce qui expliquerait en partie l'importance accrue accordée aux enjeux de la retraite.

Surtout, le modèle contribue à expliquer, mais pour une part seulement, les énigmes du comportement d'épargne. Une épargne insuffisante pour les vieux jours pourrait parfois se justifier par un risque important de mort précoce. L'investissement en rente décroît avec la concavité de $\phi$ parce que les cotisations versées accroissent le risque d'une vie courte et monotone. La concavité de $\phi$ augmente d'autant (par rapport à $\gamma$ ) l'aversion relative au risque sur la richesse qui commande les choix de portefeuille et diminue donc la demande d'actions.

Tel qu'il est spécifié, le modèle de Bommier pose cependant problème. Ses prédictions vont dans le bon sens mais ne permettent pas d'expliquer la diffusion restreinte des rentes ni la détention limitée des actions. En outre, le modèle prédit que l'aversion au risque $\gamma$ diminue avec l'âge, alors que les mesures directes de ce paramètre de préférence aboutissent uniformément au résultat contraire ( $c f$. infra). Enfin, la concavité de $\phi$ traduit, comme chez d'Alembert, une aversion à l'aléa de la durée de vie - mieux vaut avoir la certitude de vivre 70 ans que d'avoir une chance sur deux de vivre 60 ans et une chance sur deux 80 ans -, soit une préférence pour une date de mort certaine. Mais 
nous venons de voir que l'incertitude ou le flou entourant cette date fatidique faisait précisément le sel de l'existence, tandis qu'une mort programmée à une date certaine et connue s'apparente à un enfer. La contradiction vient de ce que le modèle de Bommier ne tient pas compte du caractère " endogène " de la mort : fixer à l'avance le terme de mon existence revient à une condamnation car cela me prive d'une liberté essentielle. Peut-être aussi que ce modèle, parce qu'il se contente d'introduire des préférences non séparables - mais se réfere encore au critère de l'utilité espérée appliqué à des probabilités de survie supposées connues -, ne sépare pas suffisamment le point de vue subjectif de l'épargnant de celui de la puissance publique : pour cette dernière, il est effectivement préférable que les individus vivent tous 70 ans plutôt que la moitié jusqu'à 60 et l'autre moitié jusqu'à 80 ans.

Le modèle de d'Albis et Thibault [2008] s'écarte davantage du modèle standard pour expliquer l'absence de détention des rentes par le caractère incertain (au sens de Knight) de la mort. Plus précisément, les auteurs supposent que :

- l'agent n'a qu'une idée relativement vague de ses probabilités de survie : ces dernières sont pour lui «ambigües"; - l'agent a une "aversion à l'ambiguïté» (voir le paradoxe d'Ellsberg) qui explique que ses choix ne correspondront à aucun critère d'utilité espérée, quelles que soient les probabilités (subjectives) attribuées aux événements ambigus.

Ce modèle aboutit à un résultat prometteur : même dans le cas où la rente est actuariellement neutre, bénéficiant d'un avantage maximal en termes de rendement par rapport aux placements traditionnels, il existe un seuil positif d'aversion à l'ambiguïté à partir duquel l'épargnant n'investira rien en rente viagère mais achètera au contraire une assurance temporaire décès. En dépit de ses insuffisances (l'assurance temporaire décès "libre " est peu diffusée), ce modèle accomplit donc un pas décisif, puisqu'il permet d'expliquer la désaffection pour la rente sans introduire de limites manifestes à la rationalité des épargnants. Son succès réside dans la distance élargie introduite entre le point de vue subjectif de l'épargnant par rapport à sa mort, qui fait intervenir son aversion à des probabilités de survie ambiguës, 
et celui objectif de l'assureur-vie, raisonnant sur des grands nombres et des probabilités non ambiguës.

L'enjeu que soulève le modèle de d'Albis et Thibault [2008] porte toutefois sur l'interprétation de l'aversion à l'ambiguïté pour des probabilités de survie incertaines. Pour les auteurs, les raisons demeurent purement objectives : le sujet ne peut connaitre avec précision ses probabilités de survie. Mais on peut aussi bien soutenir, ce qui n'est pas contradictoire, que le sujet ne veut pas ou ne cherche pas trop à les connaître.

Kopczuk et Slemrod [2005] sont parmi les seuls à s'être engagés dans cette dernière voie, cherchant à tirer les conséquences sur les comportements de l'épargnant du fait que ce dernier éprouve une crainte ou une angoisse irrésistible par rapport à la mort. Cette peur de la mort, inhérente à la nature humaine et donc a priori " rationnelle ", serait source pour l'individu d'un déplaisir, d'une perte d'utilité qui diminuerait lorsque les probabilités de survie anticipées augmentent. De là viendrait la tentation du déni, ou refoulement partiel (repression) de l'information reçue sur ces probabilités. Ce déni conduit à surestimer sa longévité : le taux de préférence pour le présent à l'instant $t$ n'est plus fonction du taux de mortalité objectif $q(t)$ comme dans la relation standard (8) mais d'un taux de mortalité moins élevé. L'individu est ainsi conduit à consommer trop peu sur son cycle de vie en laissant trop derrière soi. Ce dernier est donc confronté à un problème d'arbitrage entre le gain d'utilité retiré d'une longévité surestimée et la perte engendrée par des choix d'épargne sous-optimaux. En niant cette attitude spécifique à l'égard de la mort qui transite par le biais de la préférence pour le présent, les modèles existants manqueraient en fait une dimension essentielle de la rationalité de l'épargnant - ce en quoi son point de vue differe radicalement de celui du professionnel.

\section{La diversité des attitudes " rationnelles " à l'égard de la mort}

Le modèle de Kopczuk et Slemrod [2005] repose cependant sur des hypothèses alambiquées ou arbitraires et génère peu de pré- 
diction solides et encore moins testables. On peut surtout lui opposer les solutions avancées depuis l'antiquité par maintes philosophies ou morales qui, partant de prémisses comparables, conjurent autrement l'angoisse de la mort.

Prenons, à titre de simple exemple historique, le cas d'Epicure, auteur d'une morale et d'un art de vivre qui a encore de nombreux adeptes aujourd'hui : "Nous sommes nés une fois, il n'est pas possible de naître deux fois et il faut n'être plus pour l'éternité. Toi pourtant qui n'es pas de demain, tu differes de jouir! Nous consumons notre vie à force d'attendre et chacun meurt affairé." Cette sentence condense pour le philosophe André Comte-Sponville " la plus belle des sagesses " qui l'a émerveillé depuis ses 20 ans : "La mort n'est rien mais nous mourrons et il y a urgence à vivre ", autrement dit, pour nous, à consommer davantage en oubliant notre vain désir d'immortalité. Reposant également sur une préférence à l'égard du temps, la règle de comportement épicurienne adopte pourtant un point de vue opposé à celui de Kopczuk et Slemrod ; mais elle apparaît tout aussi « rationnelle »...

L'enseignement clef de la tradition philosophique tient précisément à la pluralité des attitudes possibles à l'égard de la mort : chacune découle étroitement de la conception de l'identité personnelle et du temps humain que le philosophe incriminé met en avant, du degré de cohésion ou de continuité de nos existences qui lui paraît souhaitable (Masson, [1995]). Un simple florilège nous fera mieux comprendre.

L'idéalisme rawlsien envisage un moi absolu capable de modeler à volonté ses désirs et surplombant une existence unifiée dont chaque moment compte autant, comme s'il s'agissait d'un cadre prédéfini dont la mort ne constituerait qu'une simple frontière.

Pour Heidegger, la mort revêt au contraire une importance extrême, au moins dans le mode vie " authentique " de "l'êtrepour-la-mort ». Comme pouvoir-être, le Dasein voit son horizon borné par la perspective inéluctable de la mort, qui constitue la possibilité ultime, inconditionnelle et incommunicable, celle de renoncer à soi-même, et son " pouvoir le plus propre, celui de 
s'anéantir ". Le "souci» du sujet réfléchissant sur soi comme mortel est alors poussé à son paroxysme et constitue la pierre d'angle de toute ipséité, car personne ne peut se substituer à moi pour mourir. Or cette conception édifiante, tragique ou héroïque de l'existence est sans doute ce qui le plus gêné les phénoménologues post-heideggeriens. S'élevant contre le stoïcisme ou le solipsisme (Ricoeur, [1990]) d'une existence trop préoccupée d'elle-même et "fascinée par la mort ", qui laisse le Dasein totalement esseulé, ils ont prôné une vie plus " centrée sur le présent " (Merleau-Ponty, [1945]) et davantage tournée vers autrui ; pour Lévinas [1979], notamment, la mort, par son altérité et son étrangeté radicales, empêche d'envisager une temporalité purement personnelle, fermée à l'autre.

Dans cette voie, Parfit [1984] représente sans doute la position la plus extrême. L'unité fondamentale n'est plus la "personne " sur sa vie entière mais "l'agent-aujourd'hui " : ce qu'il est rationnel de faire dépend des valeurs, croyances ou désirs actuels qui permettent de préserver son intégrité. Le comportement est alors moins autocentré : mes « moi futurs" me sont en partie étrangers et je n'ai pas à accorder un poids entier à un sosie éloigné dont je ne sais vraiment qui il sera, ni même davantage d'importance qu'à autrui. L'identité souvent indéterminée d'une personne, l'unité de sa vie comme la perspective de sa fin, ont alors moins d'importance, et Parfit prône finalement, contre le souci de soi et la recherche absolue d'ipséité, une certaine insouciance dans la conduite de son existence et un certain détachement, quasi-bouddhique, à l'égard de sa fin.

Ces quelques exemples suffisent pour notre propos : ils présentent des attitudes individuelles face à la mort très différentes, dont certaines peuvent paraître plus sympathiques que d'autres, mais qui sont toutes pareillement "rationnelles ". Au-delà de la simple donnée des probabilités mathématiques de survie, l'approche existentielle leur fera correspondre, sommairement, des profils spécifiques de la préférence temporelle pour le présent (nulle dans le cas de Rawls, élevée chez Parfit, etc.).

Le paramètre de préférence $\alpha$, qui mesure la force et l'étendue du projet actuel conférant sens et substance à l'existence, 
voit sa conception enrichie : il traduira en même temps l'attitude du sujet à l'égard de sa propre mort, qui dépendra notamment de son histoire personnelle, de son contexte situationnel. ${ }^{17}$

Les points de vue du professionnel et de l'épargnant seront d'autant différenciés, et cela d'autant plus que les projets substantiels que poursuivent aujourd'hui les épargnants et qui font leur raison de vivre peuvent n'entretenir qu'un lien ténu avec le terme de leur existence, voire en être relativement déconnectés. Même s'ils savent qu'ils "n'en ont plus pour longtemps ", des personnes âgées peuvent encore avoir des projets plutôt que d'attendre la mort en " meublant " au mieux le peu de vie qui leur reste - i.e. en se contentant d'optimiser la consommation des derniers jours compte tenu de ce qu'ils veulent laisser à leurs enfants.

Un horizon $[+\infty ; \delta(S)]$, à terme indéfini et à taux d'actualisation $\delta$ plus ou moins sensible aux probabilités de survie, peut parfaitement être rationnel. Ou encore, pour prendre un exemple caricatural, en dehors du domaine de l'épargne, qui nous fera peut-être mieux comprendre : lorsqu'il se lance dans la Recherche, Proust veut accomplir son œuvre avant que la mort ne le surprenne ; au-delà de ses considérations premières, ses probabilités objectives de survie ne l'intéressent pas.

\section{L'impossibilité de reproduire en labo- ratoire le temps long de la vie}

Les embryons de l'approche existentielle que nous venons de poser suffisent pour montrer comment cette dernière pourrait expliquer les énigmes du comportement d'épargne auxquelles le courant psycho-économique n'apporte jamais que des réponses incomplètes (voir Masson, [2010]).

Nous commencerons par envisager les implications de ce qui constitue le nœud de l'approche : sa conception enrichie 
de la préférence temporelle pour le présent, censée traduire la force et l'étendue du projet en cours du sujet et incorporer son attitude idiosyncratique à l'égard de la mort. Cette conception éclaire les échecs patents qu'ont connus les mesures expérimentales du taux d'actualisation $\delta$ et les tests du modèle de cycle de vie menés en laboratoire - échecs supposés (à tort) tenir à l'imprévoyance et à la propension à planifier limitée des épargnants.

Les caractéristiques de la subjectivité humaine et des rapports de l'individu au temps, "berceau » de cette subjectivité, et la spécificité des attitudes à l'égard de la mort semblent rendre vaine toute prétention à reproduire le temps long de la vie in vitro, dans des expériences de laboratoire destinées à tester les comportements de l'épargnant et/ou à mesurer ses préférences à l'égard du temps. L'économie expérimentale a pourtant multiplié ce type d'expériences. Pire, les tenants de l'approche psychoéconomique ont tendance à considérer sur le même plan les tests expérimentaux qui concernent les comportements à l'égard du risque - contre le modèle EU - et ceux relatifs aux choix à l'égard du temps - contre le modèle DU -, en cherchant à établir un parallèle étroit entre les " anomalies " observées dans l'un et l'autre cas par rapport au modèle standard. Du point de vue existentiel, ce parallèle constitue une escroquerie de la pensée : les tests portant sur les risques statiques, notamment, apparaissent beaucoup plus probants. Au contraire, les expériences qui supposent le transport des sujets sur de grands intervalles de temps conduisent souvent à des impasses. Nous allons en donner quelques illustrations.

\section{Des mesures expérimentales du taux de dépréciation du futur trop disparates}

Dans leur survey très complet des mesures économétriques et surtout expérimentales du taux de dépréciation du futur, Frederick et al. [2002] montrent que les estimations de $\delta$ obtenues dans la quarantaine d'essais publiés au cours des 25 années antérieures s'étagent entre $-6 \%$ et plus de $200 \%$ (!) ; en outre, cette extrême 
dispersion ne se réduit pas vraiment dans les études plus récentes, les valeurs élevées demeurant la majorité. Comment interpréter de tels écarts?

La plupart des estimations sont certes biaisées du fait qu'elles sont fondées sur une simple extrapolation de la célèbre maxime de La Fontaine : " un tiens vaut, ce dit-on, mieux que deux tu l'auras ». Mesurant davantage des taux marginaux de substitution intertemporels qu'une préférence pure pour le présent, elles sont polluées par de multiples facteurs, parfois difficiles à contrôler même en laboratoire. Si les phénomènes d'anticipation (savoring, dread...) introduisent un biais en faveur du futur, la majorité de ces facteurs perturbateurs tendent au contraire à surestimer le taux de dépréciation du futur $\delta$ : plutôt que deux demain je préfere un aujourd'hui, parce que judicieusement placé il me rapportera davantage (taux d'intérêt $r$ ) ; parce que ma consommation planifiée demain est plus faible que celle d'aujourd'hui (utilité marginale décroissante) ; par effet d'habitude ; parce que mes capacités de jouissance seront moindres demain (état de santé) ; parce que je suis contraint par la liquidité ; ou encore du fait de l'incertitude du futur : «l'un est sûr, l'autre pas» (La Fontaine).

En outre, les options alternatives étant souvent trop rapprochées, les mesures ont tendance à porter plutôt sur l'impatience à court terme $(\beta)$ ou même sur le coût du temps (utilité du loisir) : de fait, l'éventail des estimations se réduit quelque peu si l'on se limite à des horizons de choix supérieurs à un an.

Cette multiplicité des sources de biais ne suffit pas, toutefois, à expliquer la dispersion et l'instabilité des estimations. Frederick et al. [2002] reconnaissent eux-mêmes qu'un tel échec est révélateur d'un problème conceptuel dans la définition et l'interprétation du taux d'actualisation $\delta$ mais ne proposent pas vraiment de solution. Pour nous, au contraire, il est clair que les choix proposés aux sujets enquêtés, portant sur des comparaisons artificielles " entre un aujourd'hui contre deux plus tard ", ne permettent guère de rendre compte de la conception existentielle de la préférence temporelle. Cette dernière sera beaucoup mieux approchée par des questions concrètes, concernant directement 
la vie des enquêtés, qui renseignent notamment sur la nature et l'étendue des projets qui les mobilisent aujourd'hui. A partir d'une batterie de plus d'une trentaine de telles questions, concernant différents domaines de l'existence - consommation, loisir, santé, famille, travail, etc. -, nous avons ainsi établi des "scores" synthétiques, qui offrent des mesures individuelles purement ordinales mais beaucoup plus satisfaisantes de la préférence pure pour le présent sur le cycle de vie (voir Arrondel et Masson, [2007]).

\section{Un test du modèle standard du cycle de vie ou un examen de contrôle pour potaches?}

Deux tentatives de mesure expérimentale de l'élasticité de substitution $\sigma$ et du taux d'actualisation $\delta$ sont encore plus révélatrices des déboires auxquels on s'expose lorsque l'on fait l'impasse sur le contexte situationnel des individus. Leur objectif est de reconstruire directement le profil de consommation désiré par l'enquêté sur l'ensemble de son cycle de vie. Pour le réaliser, elles vont placer les sujets-cobayes dans des conditions artificielles qui correspondent aux hypothèses particulières $\mathrm{du}$ modèle standard - monde certain, marchés parfaits, connaissance totale... - en "contrôlant " en laboratoire tous les facteurs parasites ou extérieurs. Le procédé permettra de juger précisément de la validité du modèle et de la rationalité des épargnants, mais aussi d'en inférer la valeur des paramètres de préférences $\sigma(=1 / \gamma)$ et $\delta$. C'est en sorte l'idéologie du "faire le vide" : désireux de rapprocher sa discipline des sciences "dures ", l'économiste entend procéder comme en physique, où l'on vérifie par une expérience bien contrôlée que tous les corps, lourds ou légers, tombent à la même vitesse dans le vide absolu.

Kotlikoff et al. [1987] confronte ainsi des étudiants en économie de l'université de Boston à la situation suivante : "Toutes les données sont certaines et connues de vous. Vous avez 35 ans et commencez votre vie active; vous êtes célibataire et le resterez toute votre vie qui s'arrête à 75 ans. Vous prenez 
votre retraite à 65 ans. Vous ne devez pas laisser de dettes ou d'héritage derrière vous. Il n'y a pas de revenus de retraite, mais vous pouvez emprunter ou placer librement sur un marché du capital parfait, au taux d'intérêt $r$. Il n'y a pas d'inflation, il existe un seul actif patrimonial, les revenus sont exogènes..." ".

Dans ce contexte, les étudiants avaient à choisir, pour un même montant actualisé de ressources présentes et futures $W$, entre plusieurs profils de consommation selon l'âge $C(t)$, et cela selon différentes valeurs du taux d'intérêt $r$. Le test et l'identification des préférences portaient donc sur les relations (4) - la consommation ne dépend que du montant global des ressources $W$, et de manière proportionnelle - et (5) - qui relie le taux de variation de la consommation au taux d'intérêt $r$ et aux préférences $\sigma$ et $\delta$. Les réponses aux 145 questions posées devaient révéler un comportement rationnel et cohérent, guidé par un système de préférences stable. En particulier, avec une fonction d'utilité supposée isoélastique et additive, de type(1)-(3), si le sujet choisit un taux de croissance de la consommation $g_{1}$ pour $r_{1}, g_{2}$ pour $r_{2}$, $g_{3}$ pour $r_{3} \ldots$, on tire de $(5)$ :

$$
\begin{gathered}
\sigma_{12}=\left(g_{1}-g_{2}\right) /\left(r_{1}-r_{2}\right) ; \sigma_{13}=\left(g_{1}-g_{3}\right) /\left(r_{1}-r_{3}\right) \ldots \\
=>\sigma_{12}=\sigma_{13}=\ldots=\sigma
\end{gathered}
$$

pour le sujet considéré si ses choix obéissent effectivement au modèle standard. On en déduit dans ce cas la valeur du taux $\delta$ (supposé invariant selon l'âge) :

$$
\delta=r_{n}-\left(g_{n} / \sigma\right), \forall n
$$

Les auteurs américains sont, en outre, persuadés d'avoir respecté les canons de l'économie expérimentale. Ils affirment ainsi être parvenus à un degré suffisant de " parallélisme » entre les conditions de laboratoire et le monde réel - entre l'in vitro et l'in vivo - pour que l'on puisse admettre que «les sujets réagissent à une situation donnée de manière similaire dans les deux cas ». Ils pensent aussi avoir résolu le problème des incitations : pour motiver les étudiants à "révéler leurs vraies préférences " en réponse à des questions largement hypothétiques, ils ont ainsi prévu que "ceux qui exprimeraient des choix cohérents se verraient remettre un bonus à la fin de l'expérience». Ils croient 
enfin avoir intégré les possibilités d'apprentissage qu'offre la vie réelle : pour certaines questions invitant à choisir le profil de consommation de proche en proche, année par année, était indiqué, après chaque décision de l'étudiant, le montant de patrimoine disponible l'année suivante pour le familiariser avec le calcul des intérêts composés.

Nous n'épiloguerons pas sur le fait, prévisible, que l'expérience ait tourné au fiasco, avec des résultats totalement inexploitables ${ }^{18}$. C'est plus la position des auteurs qui nous intéresse : ces derniers concluent que leurs données "rejettent fortement le modèle standard du cycle de vie, à préférences homothétiques et séparables "; pire, à la question posée dans le titre de leur article : "Can people compute ?", ils répondent franchement par la négative, suggérant que la rationalité des étudiants futurs épargnants était très limitée. Ils n’ont pas vu que leur expérience, plutôt que de constituer un test de modèle, avait tous les traits d'un test de contrôle des connaissances (sur les intérêts composés, par exemple) et tournait à un pur exercice de calcul, sinon même à un examen pour potaches - ce qui en dit long sur les positions épistémologiques de certains économistes...

La vertu pédagogique de l'étude de Kotlikoff et al. [1987] vient précisément de ce qu'elle permet de démonter les mécanismes qui conduisent à une telle absurdité : transformer les choix de vie en un exercice de calcul. L'approche existentielle nous conduit à en distinguer trois types, qui participent tous de l'idéologie du "faire le vide » en arrachant les étudiants de leur contexte situationnel actuel :

- l'étude fait le vide de la "mondanéité " et de la "socialité " (Merleau-Ponty) des individus et s'adresse en fait à des sujets déracinés, désincarnés : les étudiants sont sortis de leur "monde" familier (Heidegger), de leur milieu social et familial ; des spécialistes d'économie expérimentale insistent eux-mêmes sur le rôle crucial, dans l'acquisition d'un comportement rationnel, de l'expérience et des effets de socialisation produits par la fréquentation répétée des marchés et des institutions - éléments qu'il faudrait donc reproduire dans les tests de laboratoire ; 
- elle fait le vide de la temporalité humaine puisqu'elle interroge les étudiants sur tout un parcours de vie, à partir d'une situation de référence hypothétique (35 ans, célibataire), totalement déconnectée de la leur au moment du test : comment pourraient-ils, dans un tel contexte, se sentir personnellement impliqués ou concernés par les questions posées et exprimer leur subjectivité et leurs goûts propres ? Quel jeune d'une vingtaine d'années pourrait se projeter ainsi à 35 ans en tant que célibataire (surtout s'il envisage de se marier prochainement), connaîtrait déjà les projets qui lui tiendront alors à cœur ? Loin de s'apercevoir dans une transparence absolue aux différents moments de son existence, il ne se connaît vraiment que dans le présent vécu et ne peut agir comme "une conscience thétique qui dominerait le temps de l'existence" (Merleau-Ponty) ;

- elle fait enfin le vide de la "finitude " humaine et de l'attitude de chacun à l'égard de sa propre mort, en embarquant les étudiants dans une existence programmée à l'avance jusqu'à 75 ans, en les transportant de la sorte dans un monde de science-fiction étranger au leur.

\section{Une aversion relative pour le risque $(\gamma)$ et un désir de lis- sage $(1 / \sigma)$ non corrélés...}

On pourra objecter que l'expérience de Kotlikoff et al. [1987], jamais publiée, constitue un cas isolé auquel nous accordons trop d'importance. Mais la même démarche est reprise dans l'étude référence de Barsky et al. [1997], menée sur les ménages de plus de 50 ans du panel américain Health and Retirement Survey. Un échantillon de ces derniers a été confronté à un scénario similaire, à peine moins hypothétique même s'il est adapté à des enquêtés seniors: "Supposons que vous ayez 50 ans, que vous (et votre conjoint) prendrez votre retraite à 65 ans, que votre vie s'arrêtera à 80 ans...». Là encore, les enquêtés avaient à choisir entre différents profils de consommation suivis entre 50 et 80 ans. Sous l'hypothèse de préférences séparables et isoélastiques, de type (1)-(3), les réponses obtenues en faisant varier 
le taux d'intérêt doivent permettre d'évaluer les paramètres (constants) de préférence individuels $\sigma$ et $\delta$ à partir des relations (11) et (12).

Les auteurs disposaient par ailleurs, sous la même hypothèse, d'une mesure indépendante de l'aversion relative à l'égard du risque, $\rho=\gamma$, obtenue à partir de choix de loteries portant sur le montant du revenu de cycle de vie. D'un point de vue existentiel, cette mesure est a priori plus fiable que les précédentes parce qu'elle concerne des choix statiques; bien qu'elle comporte des défauts importants (voir Arrondel et Masson, [2007]), elle contribue effectivement à expliquer certains comportements à risque recensés dans l'enquête américaine (les résultats obtenus sur données françaises sont comparables). On dispose ainsi, à partir d'estimations séparées, d'un test supplémentaire du modèle standard concernant la relation (6) $: \gamma=\sigma$.

Le module d'estimation de $\sigma$ et $\delta$, concernant 198 individus, conduit de nouveau à des résultats décevants. Les choix effectués sont souvent incohérents (l'enquêté réagissant à l'envers aux hausses du taux d'intérêt, par exemple), si bien que 82 questionnaires ne sont pas exploitables. Sur les 116 restants, les estimations de $\sigma$ sont peu stables; elles conduisent surtout à un taux d'actualisation $\delta$ négatif - le profil de consommation désiré est croissant pour un taux d'intérêt nul. Les auteurs tentent héroïquement de défendre cette conclusion surprenante en s'appuyant sur plusieurs études expérimentales du courant psychoéconomique ; or ces dernières décèlent surtout des phénomènes d'anticipation à court terme (savoring, dread...) qui introduisent bien un biais en faveur du futur mais ont peu à voir avec le taux subjectif d'actualisation $\delta$ sur le cycle de vie. Finalement le test de la relation standard $\sigma=1 / \gamma$ tourne au fiasco : la corrélation obtenue entre $\sigma$ et $1 / \gamma$ est de 0.01 , avec un écart type de 0.02 . Comme les évaluations de l'aversion au risque ont une certaine pertinence, force est d'admettre que les estimations de $\sigma$ et donc de $\delta$ sont essentiellement du bruit...

Ces échecs répétés illustrent l'impossibilité de reproduire le temps long de la vie en laboratoire. La leçon qui se dégage, encore une fois, est que les mesures expérimentales des préférences à l'égard du risque sont plus fiables que celles des préférences à 
l'égard du temps, tout particulièrement de la préférence pour le présent qui comporte des difficultés conceptuelles spécifiques.

\section{Expliquer la faible diffusion, quasi- universelle, de la rente viagère}

Rappelons les données du problème (voir Masson, [2010]). Les défauts traditionnels de la rente (antisélection, aléa moral, taux de chargement, illiquidité, non-indexation sur l'inflation, etc.) combinés à l'existence d'un motif de transmission raisonnable ne permettent pas, à eux seuls, d'expliquer la diffusion restreinte de la rente dans le modèle standard. Nous avons vu qu'une approche psycho-économique épurée, qui combinerait plusieurs éléments non standard - impatience à court terme $(\beta)$, aversion à la perte, désir de flexibilité et altruisme tempéré - pouvait encore réduire considérablement la demande, avant et après la retraite, pour cet actif qui se verrait reprocher le fait d'être, respectivement, un investissement: à trop long terme ; à fonds perdus en cas de mort précoce ; impliquant l'aliénation irréversible d'une part du capital ; à caractère anti-familial, risquant de mettre à mal les solidarités intergénérationnelles.

Le problème vient de ce que, mis bout à bout, l'ensemble de ces facteurs peut justifier des investissements restreints mais pas une demande nulle, sauf à limiter drastiquement la rationalité des épargnants : la détention de la rente viagère demeure rentable à moins d'introduire une très forte impatience à court terme, par exemple. Les éclairages complémentaires apportés par l'approche existentielle seraient donc bienvenus si l'on peut sauver ainsi la rationalité pratique des épargnants.

Levons au préalable une objection : il existe dans le cas français d'autres facteurs explicatifs, apparemment probants, de la désaffection pour la rente viagère, qui sont liés aux particularités institutionnelles et à l'histoire de notre pays (Masson, [2004]). 
La rente française serait disqualifiée aujourd'hui parce qu'elle se retrouverait " coincée " entre d'un côté, une couverture sociale généreuse et étendue (le taux de remplacement des retraites avoisine $60 \%$ en moyenne), et de l'autre une épargne assurance de longue durée (8-10 ans), fiscalement avantagée et plébiscitée. Par contre, la rente (perpétuelle mais aussi viagère) semblait bénéficier d'un véritable engouement dans la France des XVIII $\mathrm{XIX}^{\mathrm{e}}$ siècles, au point même que Mirabeau a pu s'élever contre "l'égoösme " familial des détenteurs de rente viagère. Pourquoi le placement serait-il, toutes proportions gardées, tombé en désuétude aujourd'hui ? Trois explications sont habituellement avancées : (i) celle précédente d'une rente prise en sandwich entre la retraite publique et l'épargne assurance ; (ii) l'absence d'inflation jusqu'en 1914, suivie par de multiples périodes d'inflation soutenue dont les épargnants garderaient un souvenir traumatisant ; (iii) la rente était jadis favorisée par un moindre altruisme familial, au sens où les parents s'occupaient moins du bien-être de leurs enfants, considérés plutôt, dès leur jeune âge, comme une ressource pour la famille (la loi sur l'interdiction du travail des enfants de moins de 9 ans date seulement du milieu $\mathrm{du} \mathrm{XIX}^{\mathrm{e}}$ dans les pays européens les plus développés).

Les modèles existants prennent plus ou moins en compte les deux dernières explications (inflation et motif de transmission) sans parvenir à générer une demande nulle. La première requerrait une simulation numérique précise mais pose de toute façon problème dans la mesure où elle ne vaut, à ce point, que pour notre pays. Or la détention restreinte de rentes viagères est un phénomène quasi-universel, qui exige donc une réponse d'abord théorique. Face à ce défi redoutable, l'approche existentielle fournit quelques éléments de solution portés par la préférence temporelle pour le présent $(\alpha, \delta)$, telle que nous l'avons redéfinie et enrichie.

Un temps du cycle de vie découpé en différentes phases selon les projets successifs et les coups du sort de l'existence fragmente l'horizon décisionnel et diminue d'autant le poids accordé aux avantages de la rente en termes de consommation des vieux jours. Mais les sauts de discontinuité du facteur d'actualisation temporelle $\alpha$, censés traduire ces bifurcations ou ces ruptures 
dans le cours de l'existence, ne suffiront pas en général, à eux seuls, à produire une absence de rentes dans le patrimoine ; il faudrait encore montrer comment la mobilisation des ressources sur le projet en cours, notamment avant la retraite, rend inopportun, en raison des limites à l'emprunt, le choix de soustraire une part de ses ressources pour financer dès aujourd'hui les besoins des vieux jours.

L'élément crucial concerne encore l'attitude à l'égard de la mort dont nous avons vu qu'elle pouvait prendre des formes (rationnelles) variées. Le modèle de d'Albis et Thibault [2008] constitue un premier pas dans cette voie : l'aversion à l'ambiguïté concernant une longévité incertaine (au sens de Knight) peut générer une demande nulle ${ }^{19}$; telle que nous l'avons réinterprétée, cette aversion traduit déjà une attitude vis-à-vis de la mort qui ne se limite pas à la considération des probabilités mathématiques - moyennes - de survie. Mais il est possible de concevoir, même après la retraite, des projets de vie beaucoup plus déconnectés de ces probabilités, à l'image des patrimoines professionnels qui transcendent la fin de l'existence sans forcément incorporer un motif explicite de transmission familiale.

\section{L'énigme des actions : une détention minoritaire qui ne décroît pas après la retraite}

La détention (directe ou indirecte) d'actions ne concerne au plus qu'un quart des ménages français. Comment expliquer une telle désaffection alors que l'on observe un différentiel important, à long terme, entre les taux de rendement des actions et celui des bons ou obligations - paradoxe dit de "l'énigme de la prime de risque ${ }^{20}$ ? Rappelons seulement que, même combinés à l'existence de coûts fixes de transaction, les contraintes de liquidité, le risque 
inassurable sur le revenu du travail (background risk), les modèles "non EU » d'aversion à la perte ou de persistance des habitudes, etc. ne peuvent expliquer à eux seuls une diffusion des actions aussi modeste sur l'ensemble de la population et encore limitée sur les patrimoines financiers déjà importants (voir Masson [2010]). L'approche psycho-économique a alors cherché à rendre compte du paradoxe en invoquant une rationalité toujours plus limitée des épargnants : elle en est venue à dénoncer leur manque d'information ou d'éducation financière, puis même à déplorer leur incompétence de gestion caractérisée. ${ }^{21}$

En quoi l'approche existentielle peut-elle contribuer à la résolution du paradoxe ? Nous allons voir qu'elle permet, une fois encore, de réhabiliter la rationalité pratique de l'épargnant sur son cycle de vie en prenant en compte le contexte situationnel dans lequel il se trouve ; les avatars des études psycho-économiques seront alors imputés au fait que ces dernières ignorent toute subjectivité humaine. Pour le montrer, commençons par évoquer une " expérience naturelle » où l'épargnant-investisseur est transporté, mais de manière non artificielle, dans un monde où n'interviennent plus son attitude à l'égard de la mort ni les rapports de soi à soi dans le temps.

\section{Une expérience naturelle : la gestion du fonds de réserve des retraites}

Invités à discuter, dans un comité d'experts, de l'affectation du nouveau fonds de réserve des retraites instauré «pour les générations futures ", les syndicalistes présents ont proposé de concert que $60 \%$ des sommes recueillies soient placées en actions. Les gestionnaires d'actifs ont dû temporiser et proposer seulement une part de $50 \%$ tout au plus... Comment rendre compte d'une prise de risques aussi peu représentative des comportements des épargnants français?

Une première explication, qui aura la faveur des professionnels, invoquera l'éducation et la culture financière acquises par des représentants syndicaux qui, familiarisés avec ce type de 
problème, procèderaient du coup à des choix rationnels. Mais apparemment, ces derniers ne gèrent pas de la même manière leur propre patrimoine.

Les économistes y verront plutôt l'effet de l'absence de coûts de transaction, coûts qui empêcheraient nombre d'épargnants d'investir en actions en raison de moyens trop réduits. Mais l'argumentation pèche de nouveau par son incapacité à rendre compte de l'absence d'actions dans des patrimoines financiers de taille appréciable.

L'approche existentielle propose une autre interprétation, non exclusive des précédentes. Si de manière naturelle, in vivo, le décideur est dégagé de son contexte situationnel actuel et qu'il s'agit pour lui de faire le mieux possible, objectivement, sur un horizon de très long terme (indépendant de sa propre fin), et pour un objectif collectif, celui-ci sera amené, effectivement, à faire des choix beaucoup plus en accord avec le modèle standard. Adoptant le point de vue de la puissance publique, il procèdera comme Daniel Bernoulli : visant seulement l'intérêt général, ses arbitrages dépendront d'un exercice de calcul a priori impartial, qui ne fera plus intervenir sa préférence temporelle pour le présent ni son rapport à la mort, mais seulement son attitude à l'égard du risque et, éventuellement, un taux d'actualisation objectif. Il pourra alors se permettre une prise de risque beaucoup plus élevée que sur sa propre existence : sa consommation personnelle ou celle de sa famille n'est pas en cause, ses projets les plus chers ne sont pas touchés. En outre, face à un avenir lointain difficile à prévoir, il ne doit satisfaire qu'à une obligation de moyens, autorisant des stratégies plus risquées : si les choses tournent mal, il n'éprouvera en effet que des regrets mesurés tant qu'il estimera avoir fait le mieux possible ex ante.

\section{Pourquoi la détention d'actions pourrait-elle être plus élevée chez les retraités?}

Toute autre sera la position sur son cycle de vie d'un épargnant qui ne peut pas toujours attendre 20 ou 30 ans que ses actions 
donnent leur pleine rentabilité parce qu'il doit consacrer des moyens limités à ses besoins de liquidité et à des usages plus pressants. La demande du placement dépendra alors de l'étendue temporelle des projets en cours. Si un taux $\delta$ élevé raccourcit l'horizon décisionnel et réduit donc l'intérêt de détenir un placement dont les avantages à terme sont dépréciés, les discontinuités du facteur d'actualisation $\alpha$ (correspondant à $\delta=+\infty$ ) pourraient expliquer beaucoup plus efficacement encore la non-détention d'actions.

La nature des projets poursuivis joue toutefois un rôle encore plus crucial : immobiliers ou professionnels, ils comportent souvent des phases obligées qui requièrent des efforts importants - constitution de l'apport personnel, remboursements d'emprunt - et se prêtent mal à la détention d'actions. De tels projets sont en outre risqués, conduisant par ricochet, selon le principe de substitution des risques (Gollier, [2001]), à s'exposer moins sur son patrimoine financier. De plus, cet effet de substitution des risques sera majoré si les projets en question donnent vraiment sens et substance à l'existence de l'épargnant, au point que leur échec, i.e. le fait qu'ils ne puissent être menés à bon terme, constituerait une source de regrets considérable : c'est que les risques financiers auront alors des conséquences asymétriques du fait des contraintes de liquidité.

Soit l'exemple de l'accumulation en actions d'un apport personnel destiné à l'acquisition d'une maison qui tient à cœur à toute la famille. Si les cours boursiers sont favorables, le ménage gagne certes beaucoup puisqu'il entrera plus rapidement en possession du bien convoité tout en économisant sur les loyers qu'il devrait autrement acquitter. Mais en cas de fortes moins-values, il perdrait bien davantage si jamais son projet immobilier devait avorter : renoncer à la maison de ses rêves le rendrait inconsolable. Désireux de se prémunir contre un tel risque existentiel, il sera tenté de limiter sa détention d'actions.

La conclusion générale qui se dégage de ce cas d'école est claire : du fait de la substitution des risques et de la nature $\mathrm{du}$ projet en cours, il peut exister en matière financière un écart important entre l'aversion (relative) " pure " pour le risque $\gamma$, et 
l'aversion au risque « induite " par l'exposition à d'autres risques, $\tilde{\gamma}$, parfois beaucoup plus élevée, qui gouverne la demande d'actions.

L'énigme de la diffusion limitée des actions connaît cependant des variations plus subtiles. Ainsi, la probabilité de détention a-t-elle plutôt tendance à augmenter avec l'âge et apparaît-elle plus élevée que la moyenne chez les retraités : en particulier, les retraités anciens indépendants possèdent plus souvent des actions, toutes choses égales par ailleurs, que les indépendants en activité (Arrondel et Masson, [2003]). Pourtant, leur espérance de vie est bien moindre ${ }^{22}$. Et surtout, les mesures directes, expérimentales ou empiriques, de l'aversion au risque $\gamma$ augmentent toutes avec l'âge (Barsky et al., [1997] ; Arrondel et Masson, [2007]), un résultat d'ailleurs conforme à l'intuition : les aînés ne goûtent guère les pratiques risquées. ${ }^{23}$

Comment rendre compte de ces évolutions contraires sur le cycle de vie de l'investissement en actions et de la tolérance au risque ? Oublions les raisons habituellement mises en avant - le niveau d'information financière s'accroît avec l'âge, les retraités ont davantage de temps disponible à consacrer à la gestion de ces placements, leurs pensions sont plus sûres que les revenus d'activité, etc. - pour nous concentrer sur les explications proposées par l'approche existentielle. Celles-ci tiendront à la nature des projets poursuivis. Orientés vers la consommation des vieux jours, les projets de nombreux retraités seraient le plus souvent moins accaparants et moins risqués que ceux des épargnants en activité ; et les propriétés de liquidité et de rendement des actions seraient bien adaptées à la réalisation des objectifs visés. Le projet professionnel d'un jeune indépendant qui se lance avec une faible mise de fond dans la création d'une entreprise inédite présente typiquement les caractéristiques inverses : même s'il est plutôt tolérant au risque, ce dernier préférera ne pas investir en actions. L'explication existentielle oppose ainsi $\gamma$ et $\tilde{\gamma}$ : l'aversion pure au risque $\gamma$ des actifs serait effectivement plus faible que celle des retraités, mais leur aversion au risque induite par leurs projets en cours et les phénomènes de substitution des risques, $\tilde{\gamma}$, serait au contraire souvent plus élevée. 
Finalement, pour une majorité de la population française, le projet d'investissement en actions - "boursicoter " comme on disait naguère - n'interviendrait qu'en second, un fois réalisés, ou bien écartés, des projets patrimoniaux plus fondamentaux d'ordre immobilier, familial, professionnel, etc. Il ne pourrait se déployer pleinement qu'à la condition de ne pas être trop concurrencé par d'autres objectifs plus accaparants : c'est pourquoi il convient typiquement à un célibataire endurci, sans ambition professionnelle et déjà pleinement propriétaire, qui a quelque argent de côté et peu de besoins à court terme.

L'approche existentielle met ainsi l'accent sur des déterminants individuels moins conventionnels de la demande d'actions. Elle pose, incidemment, un regard original sur une question clef, souvent escamotée dans les analyses de choix de portefeuille : la diversité d'un pays à l'autre du taux de diffusion des actions. Dans une étude concernant des données nationales rendues aussi comparables que possible, Guiso et al. [2003] recensent des taux de détention globale (directe et indirecte) des actions très disparates pour la même année 1998: $15 \%$ pour l'Italie, $23 \%$ en France, $24 \%$ aux Pays-Bas, $34 \%$ au Royaume-Uni, mais déjà $48 \%$ aux Etats-Unis ; en outre, Calvet et al. [2009] rappellent que ce même taux a augmenté de $61 \%$ à $65 \%$ en Suède entre 1999 et 2002 ! Les différences institutionnelles (en termes de fonds de pension, par exemple), les problèmes de définition et de mesure dans les comparaisons internationales, l'attractivité des placements concurrents (cas des contrats d'assurance vie en France) jouent probablement un rôle clef dans l'explication de tels écarts. Mais l'approche existentielle apporte des éclairages complémentaires liés notamment à l'importance variable des projets immobiliers et familiaux. Le statut de propriétaire bénéficie d'une image socioculturelle plus forte en France qu'ailleurs, le désir très répandu d'accès à la propriété y étant souvent interprété comme une résurgence de l'attachement à la terre dans un pays de tradition paysanne. De même, la famille (éducation et installation des enfants, transmission du patrimoine) occupe une place centrale dans des nations latines et catholiques comme la France et 
l'Italie, mais souvent plus limitée dans les mondes anglo-saxon ou scandinave.

\section{Leçons existentielles sur la rationalité pratique des épargnants}

L'approche existentielle des choix patrimoniaux de l'épargnant sur le cycle de vie tire son originalité du fait qu'elle entend prendre pleinement en compte le contexte situationnel dans lequel se trouvent les individus : ces derniers se déterminent en fonction de leurs projets en cours, ceux qui donnent aujourd'hui sens et substance à leur existence ; leur horizon temporel est fragmenté par les ruptures ou bifurcations, subies ou choisies, qui découpent leur cycle de vie en autant de phases successives ; leur rapport à la mort n'est pas seulement régi par leurs probabilités mathématiques de survie, qu'elles soient correctement anticipées ou non.

Cette approche existentielle répond-elle aux énigmes que posent les comportements de l'épargnant ? Notre étude répond par l'affirmative bien qu'elle reste exploratoire : si elle dénonce les insuffisances des modèles existants elle ne propose pas de formalisation alternative précise. Elle demeure par ailleurs trop " académique » en ce qu'elle ne dégage pas les implications que l'on peut tirer de notre approche du côté de l'offre : quels produits d'épargne nouveaux pourrait-on ainsi mettre en place qui seraient mieux adaptés à la rationalité pratique et aux besoins spécifiques des épargnants?

Quoi qu'il en soit, le point de vue existentiel n'a pas vocation à remplacer les approches précédentes, dont il ferait table rase, mais seulement à proposer des éclairages complémentaires que ces dernières ignorent. Les analyses que nous avons menées montrent bien que ce dernier n'aboutit à des implications intéressantes qu'en combinant les apports existentiels à d'autres 
éléments, que ceux-ci soient standard (contraintes de liquidité, substitution des risques) ou hérités du courant psycho-économique - concernant alors aussi bien le temps (impatience à court terme, préférences non additives) que les comportements face à l'incertain (aversion à la perte, préférence pour la flexibilité, aversion à l'ambiguïté des probabilités de survie, etc.). Cependant, l'approche existentielle modifie en profondeur le point de vue sur l'épargnant et sur sa rationalité, en contestant la vulgate en vigueur chez les économistes comme chez les professionnels de la finance ou de l'assurance. Nous en tirerons trois conclusions générales.

\section{La rationalité de l'épargnant est moins limitée qu'il n'y paraît}

L'épargnant (français) ne semble pas aussi "myope » ou " timoré " que ne le pensent et ne le déplorent les tenants du courant comportemental ou psycho-économique.

Pour dénoncer la rationalité limitée d'épargnants trop myopes - incapacité à planifier, imprévoyance, incohérence temporelle des choix, épargne inadéquate pour la retraite, etc. - ce courant s'appuie pour une large part sur les résultats négatifs de tests expérimentaux qui visent à reproduire en laboratoire le temps long de l'existence. Plaçant les sujets-cobayes dans une sorte "d'intra-temporalité " artificielle mal contrôlée, ces tests font fi de toute subjectivité humaine et sortent les individus de leur contexte situationnel du moment ; aussi transforment-ils les choix de vie des sujets en un exercice ou un examen de calcul... où ils distribuent effectivement de mauvaises notes. Nous avons souligné la réticence des économistes concernés à reconnaître, même dans les études les plus récentes, l'inanité de telles expériences qui ne peuvent servir ni à tester le modèle du cycle de vie, ni à apprécier le degré de rationalité des épargnants.

Une réinterprétation existentielle du facteur d'actualisation temporelle $\alpha$ permet par ailleurs de considérer les sauts de discontinuité qu'enregistre ce paramètre de préférence aux nœuds subis ou désirés du cycle de vie comme des " petites morts " suivies 
de "petites renaissances ». Le passage d'un nœud génère ainsi des myopies temporaires mais néanmoins rationnelles (temporellement cohérentes) : l'horizon semble par trop se rétrécir avant l'échéance fatidique pour s'ouvrir à nouveau après coup. Ces phénomènes de morcellement de l'horizon et le rapport spécifique de l'épargnant à la mort, que ne déterminent pas entièrement ses probabilités mathématiques de survie, permettent d'expliquer la diffusion limitée de la rente viagère sans recourir, comme le courant psycho-économique, à des biais de rationalité ad hoc.

Les trois quarts des ménages français qui ne détiennent pas d'actions ne sont pas forcément des épargnants trop timorés. Leur comportement pourrait s'expliquer par un écart important entre l'aversion pure au risque $\gamma$ et celle $\tilde{\gamma}$, induite par le contexte situationnel. On sait qu'exposé à un risque inévitable et inassurable (background risk) sur son revenu ou sa santé, le sujet standard tempère les risques qu'il contrôle, si bien que l'aversion $\tilde{\gamma}$ induite par cette exposition est plus élevée que l'aversion ex ante $\gamma$ (Gollier, [2001]). Un phénomène analogue de substitution des risques a lieu, dans une perspective dynamique, lorsque l'épargnant est confronté à un risque de nature existentielle, concernant la réussite du projet qu'il a à cœur. Seulement, le phénomène connaîtra une toute autre ampleur ( $\tilde{\gamma}$ plus élevée) si la non-réalisation du projet de ses rêves entraîne pour l'épargnant des regrets considérables : afin de s'éviter de tels regrets, ce dernier adoptera une gestion financière beaucoup plus prudente. Autrement dit, la probabilité de détenir des actions dépendra de la nature du projet poursuivi, du risque d'échec de ce projet, mais aussi des regrets anticipés dans ce cas - dont on sait qu'ils constituent une donnée essentielle jouant négativement dans toute prise de risque.

\section{Le développement de l'éducation financière n'aura pas for- cément les résultats attendus}

Bien qu'elle soit invoquée comme une panacée par le courant psycho-économique comme par les professionnels, l'éducation 
financière ne constitue pas toujours un remède efficace contre les " anomalies» du comportement des épargnants : épargne inadéquate pour la retraite, détention minoritaire des actions (en France), diffusion restreinte des rentes viagères.

Supposons en effet que les facteurs mis en avant par l'approche existentielle, combinés aux imperfections des marchés du capital (contraintes de liquidité, coûts de transaction) et à des préférences non standard (aversion à la perte, désir de flexibilité), jouent un rôle déterminant dans l'explication de ces dites anomalies. Améliorer l'éducation financière des agents pourrait bien n'avoir alors qu'une efficacité limitée. La littérature économique de plus en plus vaste consacrée à ce sujet (voir par exemple Lusardi, [2009ed]), qui s'intéresse en priorité à la sous-épargne chronique des ménages américains, conclut plutôt dans ce sens : elle ne met tout au plus en évidence que des effets modestes de la formation financière - financial literacy - des épargnants.

Cette inefficacité n'est pas surprenante. L'acquisition de l'éducation financière semble d'abord résulter d'un processus long de transmission spécifique par le milieu familial : avoir (eu) des parents prévoyants, prudents, ou altruistes, a des effets positifs et significatifs sur (respectivement) la prévoyance, la prudence ou l'altruisme de l'individu, qui s'avèrent sensiblement plus importants que ceux de l'éducation religieuse, de l'origine sociale ou du diplôme ${ }^{24}$; de même, le fait que les parents sont ou étaient assurés sur la vie apparaît le facteur explicatif le plus probant de la détention d'assurance vie et d'assurance décès; le résultat est analogue, et presque aussi important, pour la possession de valeurs mobilières (Arrondel et al. [2003], chap. 3). En outre, vouloir améliorer l'éducation financière apparaît illusoire pour les produits dédiés à la retraite, beaucoup trop complexes. Le cas du Perp (cantonnement, taux d'actualisation nul pour le premier versement de la rente, changement de table de mortalité, déduction des cotisations et taxation de rentes non indexées sur l'inflation, etc.) est à cet égard exemplaire : les conseillers financiers, eux-mêmes peu au fait des subtilités du produit, se contentent de communiquer sur la nécessité d'un complément retraite et l'existence d'avantages fiscaux (Masson, [2009]). 
De fait, les partisans d'un développement de l'épargne privée pour les vieux jours, qui recourent moins à l'éducation financière stricto sensu qu'à des incitations contraignantes ou manipulatrices (nudging), reconnaissent implicitement que le rêve de produire un "épargnant nouveau " est une chimère. Sous couvert d'un " paternalisme libertaire " qui offre, comme solution par défaut, l'inscription automatique sur un programme de base plutôt que la non participation au plan d'épargne retraite (Benartzi et Thaler, [2004]), ils en viennent souvent à imposer l'affiliation quasi-obligatoire. Le programme singapourien Eldershield de financement privé (dès l'âge de 40 ans) de l'assurance dépendance, ne laissait ainsi qu'un délai de trois mois pour refuser toute participation; il s'est révélé particulièrement efficace, puisque seuls $14 \%$ de la population n'étaient pas couverts par le programme en 2006.

\section{Pour une gestion intermédiée mais souple de l'épargne longue}

Enfin, une gestion intermédiée de l'épargne longue apparaît souvent préférable, à condition de privilégier davantage les intérêts de l'épargnant et d'être mieux adaptée à ses besoins et projets spécifiques. La participation au plan d'épargne pourrait être encouragée par des incitations diverses, mais ne serait pas a priori rendue obligatoire. Commentons chaque point.

Pour l'approche existentielle, même si les épargnants sont plus rationnels qu'on ne le croit, il conviendrait de confier la gestion de l'épargne longue ou de retraite à un intermédiaire financier (ou un gestionnaire public) "bien intentionné ». Ce n'est pas que le professionnel soit forcément plus compétent ou mieux informé que l'épargnant, ni qu'il fasse montre d'une rationalité toujours supérieure dans ses choix. Mais, à l'instar de Daniel Bernoulli ou du gestionnaire du fonds de réserve des retraites que nous avons pris pour exemple, il peut adopter la position objective et de long terme requise pour faire fructifier au mieux le capital qui lui est confié, prendre le point de vue collectif du grand nombre et des probabilités mathématiques de 
survie, considérer même des horizons plus lointains intégrant le bien-être des générations futures. Affranchi ou presque de tout contexte situationnel, il va se déterminer en fonction d'une aversion au risque proche de son aversion pure et d'une préférence temporelle faible et sans à-coup.

Cette conclusion paradoxale d'une approche existentielle respectueuse de la rationalité pratique de l'épargnant vient de ce qu'elle éloigne radicalement les points de vue de l'épargnant et du gestionnaire en reconnaissant que la subjectivité humaine est mal adaptée à la constitution précoce et à la gestion efficace de l'épargne pour les vieux jours. Mais cet écart crée une difficulté : une gestion professionnelle, effectuée dans l'intérêt collectif des épargnants, doit en même temps être sommairement adaptée aux besoins et aux projets propres de chacun, besoins et projets dont les caractéristiques observables de sa situation actuelle donnent une idée. C'est ainsi que l'on peut comprendre les recommandations de Stiglitz [2007], visant à introduire dans les plans de retraite américains un système d'options par défaut qui seraient décontractées (customized) non seulement selon l'âge de l'épargnant, mais aussi selon sa situation familiale, un indicateur rudimentaire de ses préférences à l'égard du risque et du temps, etc.

Nous avons nous-mêmes proposé un produit d'épargne pour les vieux jours, le viager intermédié partiel, qui ne porterait que sur une part fixée du logement et serait offert par un intermédiaire financier (Masson, [2009]). Le produit conviendrait mieux à la rationalité pratique des épargnants : les enfants resteraient notamment " cohéritiers " avec la société d'assurance, et il pourrait être couplé à une assurance dépendance (la rente versée augmenterait fortement en cas de perte d'autonomie). Son inconvénient majeur serait, au rebours de la tendance actuelle, de faire porter la majorité des risques par l'assureur. Pour l'approche existentielle, cet inconvénient n'est pas forcément rédhibitoire (parce que l'épargnant est déjà soumis à des risques de longue portée sur son existence personnelle, dus à des coups du sort ou à l'échec de ses projets de vie); mais bien sûr, d'autres points de vue sont possibles. 
Enfin, l'idée de plus en plus en vogue, avertie des échecs de l'éducation financière, de rendre la participation à certains plans d'épargne ou l'assurance privée dépendance obligatoires (comme dans le cas de la voiture ou du logement) ne se justifie pas aisément dans un cadre existentiel qui accorde un poids important au point de vue subjectif, personnel de l'épargnant : pour ce dernier, l'obligation peut s'avérer particulièrement dommageable quand bien même elle se justifierait pleinement au plan théorique ou collectif. On est ainsi ramené au débat exemplaire entre Bernoulli et d'Alembert sur l'inoculation de la petite vérole : le premier, suivi par tout un courant scientifique et politique, s'appuyant sur le calcul économique et probabiliste pour justifier de la rendre obligatoire ; le second, minoritaire et mal compris, proposant seulement de l'encourager...

L'auteur remercie Luc Arrondel, Pierre Pestieau et un commentateur anonyme pour la qualité de leur lecture et la pertinence de leurs remarques sur une version antérieure de ce texte.

André Masson est directeur de recherche au CNRS, directeur d'études à l'EHESS, membre du PSE (Paris Jourdan Sciences économiques), et chercheur associé à la chaire "Transitions démographiques, transitions économiques" (Fondation du risque).

Adresse : PSE Paris Jourdan Sciences économiques, Ecole Normale Supérieure, 48 bd Jourdan, 75014 Paris.E-mail : masson@pse.ens.fr 


\section{Notes}

1. Que ces philosophies (post-kantiennes) relèvent de la phénoménologie continentale (Husserl, Heidegger, Merleau-Ponty ou Ricoeur), des courants analytiques anglo-saxons (Parfit, Williams), ou du « rationalisme » à la Bachelard.

2. Pour plus de détails, voir Masson [1995 et 2000].

3. $C f$. Williams [1981] qui s'élève contre " cette perspective [qui] omet un fait crucial, à savoir que la continuation et la taille du rectangle dépendent de soi ».

4. Dans un autre contexte, Bachelard [1932] reprend les mêmes idées : « c'est en tendant notre conscience vers un projet plus ou moins rationnel que nous trouverons vraiment la cohérence temporelle fondamentale qui correspond pour nous à la simple habitude d'être » (p. 73). Le concept clef d'habitude joue le rôle de celui de la répétition chez Heidegger : « ce qu'il peut y avoir de permanent dans l'être est l'expression, non d'une cause immobile et constante » (p. 23), ni la trace laissée par un passé révolu, mais le produit d'une " habitude ", soit la volonté active et créatrice de se répéter soi-même, "l'éternelle reprise » des possibilités que l'on se transmet (p. 79-81). Dans un temps discontinu ne dure en effet que ce qui recommence, ou mieux ce « qui a des raisons de recommencer» (Bachelard, [1936]).

5. Sa conception monadique de «l'êtrepour-la-mort », sa vision stoïcienne désespérée du destin «authentique» d'un Dasein esseulé, (primant toute destinée collective) sa notion de communauté ou de peuple, etc.
6. Le choix par Achille d'une vie courte et brillante plutôt que longue et monotone (révélant pour nous une forte préférence pour le présent) relève certes de sa subjectivité ou de sa liberté la plus propre mais correspond en même temps à ce que l'on pouvait attendre d'un guerrier achéen ivre de gloire, appelé à transcender sa peur de la mort.

7. Soit en quoi le moi en $t$ est semblable ou étranger («autre ») au moi d'aujourd'hui. Ce formalisme ne doit pas être confondu avec la conception scalaire de l'identité au cours du temps adoptée par Parfit [1984] au plan philosophique, qui a été très critiquée pour ses postulats matérialistes : voir notamment Williams [1981] et Ricoeur [1990].

8. Si l'on suppose qu'une prévoyance élargie a un coût, $\alpha$ peut même dépendre (positivement) du montant de la richesse courante $A(t)$ comme le proposent Becker et Mulligan [1997], ce qui introduit une non-homothétie dans des préférences qui restent isoélastiques - relation (3).

9. Contre «l'illusion biographique» qui ferait de la vie «un tout, un ensemble cohérent et orienté », associée à un sujet « dont la constance n'est sans doute que celle d'un nom propre», Bourdieu [1986] rappelle l'emploi proustien de l'article défini devant la personne pour suggérer précisément les phases successives de l'existence : le Swann de Buckingham Palace, l'Albertine d'alors.

10. $C f$. Masson [1995] : chaque trajectoire de vie correspond à un profil temporel donné du facteur d'actualisation $\alpha(t)$.

11. De même, la dépendance dite «lourde» à l'âge élevé, qui marque une 
altérité profonde du moi, se traduit $a$ priori par une baisse importante de $\alpha$ (le sujet se reconnaît difficilement dans cet autre lui-même qu'il serait alors devenu) : mais l'horizon de l'épargnant intégrera encore le fait que cette dépendance ne constitue qu'un risque, la probabilité de la connaître étant évaluée à $20 \%$ environ à l'entrée en retraite.

12. Voir la relation (4). Intuitivement, si $k=1 / 4$ par exemple, je consomme l'année actuelle $25 \%$ d'un revenu d'aubaine (windfall gain) ; avec une consommation constante, j'aurai donc entièrement consommé ce revenu au bout de 4 ans.

13. On notera que la perspective du mariage ou du métier salvateur, entraînant un baisse ultérieure de $\alpha$, raccourcit encore l'horizon et pousse d'autant plus le jeune homme à se droguer... à ses risques et périls si cette perspective ne se réalise pas ou constitue une simple chimère, voire un prétexte.

14. Il en sera de même si l'individu mène une vie austère - quitte à laisser ses biens à une œuvre caritative - parce qu'il se rendrait compte, tout d'un coup, que sa vie précédente ne rimait à rien.

15. Référence que nous devons à Luc Arrondel.

16. La formule standard (7) suppose au contraire une neutralité par rapport à ce risque sur la durée de vie.

17. Le profil de la préférence discontinue $\alpha$ dépendra, plus généralement, de l'attitude du sujet vis-à-vis des « petites morts » qui jalonnent son cycle de vie : on peut par exemple craindre ou au contraire espérer l'arrivée de la retraite.

18. Les auteurs ont malgré tout tenté d'estimer économétriquement les valeurs moyennes des paramètres $\delta$ et $\sigma$ sur l'ensemble de l'échantillon, les évalua- tions plausibles obtenues attestant selon eux du sérieux minimum des réponses...

19. Pour traduire le fait que les nœuds de l'existence, tels le mariage ou la retraite, constituent, en tant que " petites morts ", des sauts dans l'inconnu, on pourrait rendre la préférence temporelle $\alpha$ ambiguë à ses points de discontinuité : le rejet de la rente viagère pourrait alors plus facilement s'expliquer par l'existence d'un horizon décisionnel morcelé par les bifurcations ou ruptures du cycle de vie.

20. Le différentiel apparaît cependant moindre en France où son existence est même contestée. Pour Tanay [2002], un investisseur qui aurait en 1914 placé 1000 francs en actions aurait vu son capital multiplié par 31 (en valeur réelle) à la veille de l'an 2000, alors qu'il n'aurait récupéré que 400 francs s'il avait acheté des obligations. Pour Le Bris et Hautcoeur [2008], qui utilisent d'autres pondérations pour le calcul des indices boursiers de 1854 à 2007, les actions auraient enregistré un rendement réel positif au $\mathrm{XIX}^{\mathrm{e}}$, mais négatif au $\mathrm{XX}^{\mathrm{e}}$ siècle (en raison de l'inflation et des deux guerres mondiales), ce qui réduirait à rien le paradoxe sur cette période. Bien sûr, l'investisseur aurait pu jouer sur une diversification internationale (américaine) de son portefeuille, plus favorable...

21. Une explication possible - encore mal élucidée - de la diffusion limitée des actions et du différentiel important du taux de détention entre la France et les Etats-Unis, notamment, proviendrait des anticipations subjectives des ménages relatives au rendement et au risque des actions à échéance donnée (5 ou 10 ans par exemple). Ces anticipations seraient nettement plus pessimistes en France qu'outre-Atlantique, et sensiblement inférieures à ce que suggère l'expérience passée concernant l'évolution des cours boursiers dans 


\section{André Masson}

notre pays. La crise aurait par ailleurs entraîné chez nous une baisse uniforme des estimations de rendement moyen (Arrondel et Masson, [2010]), contrairement au cas des Etats-Unis où les évolutions individuelles des anticipations, à la hausse ou à la baisse, seraient beaucoup plus contrastées (Hudomiet et al., [2009]).

22. Même si cette espérance de vie plus faible est partiellement compensée, quant à l'horizon décisionnel, par un taux d'actualisation temporelle $\delta$ moins élevé chez les retraités (voir Arrondel et Masson, [2007]).
23. Sur données françaises en coupe instantanée (Insee [1998], Sofres [2002, 2007] et [2009]), les diverses mesures de l'attitude à l'égard du risque concluent toutes que cette dernière augmente significativement avec l'âge. En panel, Arrondel et Masson [2009] obtiennent le même résultat (entre [2002 et 2007]), ainsi que Sahm [2008]) sur données américaines (HRS).

24. Sur la transmission des préférences, voir Arrondel et Masson [2007], chap. 10, et surtout Arrondel [2009]. 


\section{Références}

L. Arrondel [2009], "Mon père avait raison" : la transmission des valeurs entre les générations, Revue française d'économie, XXIV (1), pp. 157-205.

L. Arrondel et A. Masson [2003], Stockholding in France, in Stockholding in Europe, L. Guiso, M. Haliassos et T. Jappelli eds., Palgrave Macmillan Publishers, Hampshire, pp. 75-109.

L. Arrondel et A. Masson [2007], Inégalités patrimoniales et choix individuels. Des goûts et des richesses, Economica, Paris.

L. Arrondel et A. Masson [2009], How to Measure Risk and Time Preferences of Savers? France : 1998, 2002 and 2007, mimeo, PSE-Jourdan, Paris.

L. Arrondel et A. Masson [2010], French Savers in the Economic Crisis: What has Changed? Variation of Preferences or Simple Adaptation to a New Environment, mimeo, PSE-Jourdan, Paris.

L. Arrondel, A. Masson et P. Pestieau [2003], Epargne, assurance vie et retraite, Éditions Economica, Paris.

G. Bachelard [1932], L'intuition de l'instant, Gonthier, Paris.

G. Bachelard [1936], La dialectique de la durée, PUF, Paris.

R.B. Barsky, M.S. Kimball, F.T. Juster et M.D. Shapiro [1997], Preference Parameters and Behavioral Heterogeneity : An Experimental Approach in the Health and Retirement Survey, Quarterly Journal of Economics, 112 (2), pp. 537-580.

G.S. Becker [1992], Habits, Addictions, and Traditions, Kyklos, 45 (3), pp. 327 346.

G.S Becker. et C.S. Mulligan [1997], On the Endogenous Determination of Time
Preference, Quarterly Journal of Economics, 112 (3), pp. 729-758.

G.S. Becker et K.M. Murphy [1988], A Theory of Rational Addiction, Journal of Political Economy, 96 (4), pp. 675-700.

S. Benartzi et R.H. Thaler [2004], Save More Tomorrow : Using Behavioral Economics to Increase Employee Saving, Journal of Political Economy, 112 (1), S164-186.

A. Bommier [2008], Mortality, Time preference and Life-cycle Models, mimeo, Gremaq, Toulouse.

P. Bourdieu [1986], L'illusion biographique, Actes de la Recherche en Sciences Sociales, 62-63, pp. 69-72.

L.E Calvet, J.Y. Campbell et P. Sodini [2009], Measuring the Financial Sophistication of Households, American Economic Review, (Papers and Proceedings), 99 (2), pp. 1-5.

H. d'Albis H. et E. Thibault [2008], Ambiguous Life Expectancy and Optimal Annuitization, mimeo, Toulouse School of Economics.

I. Fisher [1930], The Theory of Interest, Macmillan, New-York.

S. Frederick, G. Loewenstein et T. O'Donoghue [2002], Time Discounting and Time Preference : a Critical Review, Journal of Economic Literature, 40, pp. 351-401.

M. Friedman [1963], The Concept of Horizon in the Permanent Income Hypothesis, in Measurement in Economics : Studies in Mathematical Economics and Econometrics, C.F. Christ, M. Freidman eds., Stanford University, Stanford, pp. 1-22. 
L. Guiso, M. Haliassos et T. Jappelli [2003], Stockholding : A European Comparison, in Stockholding in Europe, L. Guiso, M. Haliassos et T. Jappelli eds., Palgrave Macmillan Publishers, Hampshire, pp. 3-29

M. Heidegger [1927], Sein und Zeit, traductions françaises Etre et temps partielle (H. Corbin in Qu'est-ce que la métaphysique ?) 1951 ; totale, (F. Vezin), Gallimard, 1987.

M. Heidegger [1946], Lettre sur l'humanisme, Aubier-Montaigne, Paris.

P. Hudomiet, G. Kezdi et R.J. Willis [2009], Stock Market Crash and Expectations of American Households, mimeo, Michigan University.

W. Kopczuk et J. Slemrod [2005], Denial of Death and Economic Behavior, NBER Working Paper 11485, reproduit in Advances in Theoretical Economics, 5 (1), Article 5.

L.J. Kotlikoff, S. Johnson et W. Samuelson [1987], Can people Compute? An Experimental Test of the Life-Cycle Consumption Model, NBER Working Paper 2183.

E. Lévinas [1979], Le Temps et l'Autre, Fata Morgana, Paris.

D. Le Bris et P.C. Hautcoeur [2008], Challenge to Triumphant Optimists? A New Index for the Paris Stock-Exchange (1854-2007), mimeo, Paris School of Economics.

A. Lusardi [2009ed.], Overcoming the Saving Slump. How to Increase the Effectiveness of Financial Education and Saving Programs, Chicago Press, Chicago.

A. Masson [1995], Préférence temporelle discontinue, cycle et horizon de vie, in Le modèle et l'enquête, L.A. Gérard-Varet et J.C. Passeron eds., EHESS, Paris, pp. 325-400.
A. Masson [2000], L'actualisation du futur, Le genre humain, $35,\left(\mathrm{n}^{\circ}\right.$ spécial: Actualités du contemporain), pp. 197244.

A. Masson [2004], Pourquoi la rente viagère ou la vente en viager sont-elles si peu diffusées?, Risques, 59, pp. 60-66.

A. Masson [2009], Le viager intermédié partiel (VIP), produit d'avenir?, Risques, 79, pp. 103-112.

A. Masson [2010], La vie pour l'épargnant ne se réduit pas à un exercice de calcul. 1 . Les impasses des approches du cycle de vie standard et psycho-économique, Revue française d'économie, XXV (1), (juillet 2010), pp. 117-173.

M. Merleau-Ponty [1945], Phénoménologie de la perception, TEL, Gallimard, Paris.

D. Parfit [1984], Reasons and Persons, Oxford University Press, Oxford.

J. Rawls [1971], A Theory of Justice, Harvard University Press, Cambridge.

P. Ricoeur [1990], Soi-même comme un autre, Seuil, Paris.

C. Sahm [2008], How Much Does Risk Tolerance Change?, Finance and Economics Discussion Series, 2007-66, Federal Reserve Board, Washington.

J. Stiglitz [2007], Recognizing the Risks of Securitization, supplément du Financial Times : Long-Term Investment, 29 novembre, pp. 14-15.

A. Tanay [2002], Les actions plus rémunératrices que les obligations et l'or au $X X^{e}$ siècle, Insee Première, $\mathrm{n}^{\circ} 827$.

L. Von Mises [1949], Human Action, Yale University Press, Yale.

B.A.O. Williams [1981], Persons, Character and Morality, in Moral Luck, Cambridge University Press, pp. 1-19 (1re éd. 1976). 
André Masson 57

M.E. Yaari [1965], Uncertain Lifetime, Life Insurance, and the Theory of the
Consumer, Review of Economic Studies, 32, pp. 137-160. 